OPEN ACCESS

Edited by:

Pingyi $X u$,

First Affiliated Hospital of Guangzhou Medical University, China

Reviewed by:

Zhong Pei,

Sun Yat-sen University, China

David J. Koss,

Newcastle University, United Kingdom

${ }^{*}$ Correspondence:

Xiaojun Lu

luxiaojun5215@163.com

Guanghui Wang

wanggh@suda.edu.cn

tThese authors have contributed equally to this work

Received: 07 April 2021

Accepted: 10 May 2021

Published: 03 June 2021

Citation:

Ren H, Zhai W, Lu X and Wang G

(2021) The Cross-Links

of Endoplasmic Reticulum Stress,

Autophagy, and Neurodegeneration

in Parkinson's Disease.

Front. Aging Neurosci. 13:691881

doi: 10.3389/fnagi.2021.691881

\section{The Cross-Links of Endoplasmic Reticulum Stress, Autophagy, and Neurodegeneration in Parkinson's Disease}

\author{
Haigang Ren ${ }^{1,2 t}$, Wanqing Zhai ${ }^{1+}$, Xiaojun $\mathrm{Lu}^{1 *}$ and Guanghui Wang ${ }^{1,2 *}$ \\ ${ }^{1}$ Department of Neurology, Center of Translational Medicine, Taicang Affiliated Hospital of Soochow University, The First \\ People's Hospital of Taicang, Suzhou, China, ${ }^{2}$ Jiangsu Key Laboratory of Translational Research and Therapy \\ for Neuropsychiatric Disorders, Department of Pharmacology, College of Pharmaceutical Sciences, Soochow University, \\ Suzhou, China
}

Parkinson's disease (PD) is the most common neurodegenerative movement disorder, and it is characterized by the selective loss of dopaminergic (DA) neurons in the substantia nigra pars compacta (SNpc), as well as the presence of intracellular inclusions with $\alpha$-synuclein as the main component in surviving DA neurons. Emerging evidence suggests that the imbalance of proteostasis is a key pathogenic factor for PD. Endoplasmic reticulum (ER) stress-induced unfolded protein response (UPR) and autophagy, two major pathways for maintaining proteostasis, play important roles in $\mathrm{PD}$ pathology and are considered as attractive therapeutic targets for PD treatment. However, although ER stress/UPR and autophagy appear to be independent cellular processes, they are closely related to each other. In this review, we focused on the roles and molecular cross-links between ER stress/UPR and autophagy in PD pathology. We systematically reviewed and summarized the most recent advances in regulation of ER stress/UPR and autophagy, and their cross-linking mechanisms. We also reviewed and discussed the mechanisms of the coexisting ER stress/UPR activation and dysregulated autophagy in the lesion regions of $\mathrm{PD}$ patients, and the underlying roles and molecular crosslinks between ER stress/UPR activation and the dysregulated autophagy in DA neurodegeneration induced by PD-associated genetic factors and PD-related neurotoxins. Finally, we indicate that the combined regulation of ER stress/UPR and autophagy would be a more effective treatment for PD rather than regulating one of these conditions alone.

Keywords: Parkinson's disease, ER stress, UPR, autophagy, $\alpha$-synuclein, cross-link

\section{INTRODUCTION}

Parkinson's disease (PD), the most common neurodegenerative movement disorder, affects approximately $1 \%$ of the population over 60 years old, and its incidence dramatically increases to approximately $5 \%$ in the population greater than 85 years of age ( $\mathrm{Li}$ and Le, 2020; Wang et al., 2020b). PD is a chronic, irreversible, and complex neurodegenerative disease with several typical motor impairments including resting tremor, bradykinesia, rigidity, and postural imbalance. These 
are caused by the selective and progressive loss of dopaminergic (DA) neurons in the substantia nigra pars compacta (SNpc) and the deficiency of dopamine release in the striatum (Poewe et al., 2017). In addition to DA neuron loss, the presence of intracellular inclusions called Lewy bodies (LBs) with an accumulation of protein aggregates including $\alpha$-synuclein ( $\alpha$-SYN), and abnormal dystrophic neurites termed Lewy neurites (LNs) in surviving DA neurons, are also the major hallmarks of PD pathology (Kalia and Lang, 2015). Recently, great achievements have been made in understanding PD pathogenesis, which may contribute to developing more optimal strategies for PD treatment.

The mechanisms of DA neuron damage in PD include a variety of cellular processes such as $\alpha$-SYN aggregates, mitochondrial damage, oxidative stress, calcium homeostasis dysfunction, axonal transport disruption, and neuroinflammation injury, and also involve aging, and environmental and genetic factors (Shulman et al., 2011). Emerging evidence suggests that these disadvantages can perturb the balance of cellular homeostasis (proteostasis) in PD (Lehtonen et al., 2019). Endoplasmic reticulum (ER) stress-induced unfolded protein response (UPR) and autophagy, two major pathways that respond to an imbalance in cellular homeostasis, play particularly important roles in the pathology of neurodegenerative diseases including PD (Karabiyik et al., 2017; Costa et al., 2020).

Endoplasmic reticulum stress, UPR, and autophagy have become important targets for alleviating the damage of DA neurons, and they provide attractive clues for the treatment of PD (Moors et al., 2017; Martinez et al., 2019). However, UPR and autophagy are both related and independent cellular processes. Both the effects of their cross-talk and their unique mechanisms in PD must be considered in order to find an appropriate therapeutic target for PD treatment or to provide the basis for combination medication. In this review, we summarize the most recent advances in ER stress, UPR, autophagy, and their interactions in PD pathogenesis, and how to alleviate the toxic effects of cellular homeostasis imbalance in PD by targeting ER stress, UPR, and autophagy.

\section{ER STRESS AND UPR}

The ER is essential for cellular homeostasis. The ER organelles have a variety of important functions that are necessary for protein synthesis, protein quality control, $\mathrm{Ca}^{2+}$ homeostasis, and lipid and carbohydrate metabolism (Wang and Kaufman, 2016; Ghemrawi and Khair, 2020). Under physiological states, the ER uses the resident chaperone molecules to ensure the proper folding of newly synthesized proteins, and also can identify misfolded proteins through quality control mechanisms and employ proteasomes to implement ER-associated degradation (ERAD) (Walter and Ron, 2011). When the protein-folding capacity of the ER is saturated under various pathological conditions, such as aberrant aggregation of misfolded proteins or mutant proteins, accumulation of exogenous viral proteins, fluctuation of ER $\mathrm{Ca}^{2+}$ stores, perturbation of ATP levels, presence of environmental toxins, or occurrence of metabolic dysfunctions, the cells sense the ER stress and subsequently initiate an adaptive response referred to as UPR. This in turn attempts to alleviate ER stress by enhancing the protein-folding capacity and reducing the general synthetic load to restore ER homeostasis and maintain cell survival (Xu et al., 2012; Rashid et al., 2015).

The UPR is controlled by three ER-resident sensors: inositolrequiring kinase $1 \alpha$ (IRE1 $\alpha)$, protein kinase RNA-activated (PKR)-like ER kinase (PERK), and activating transcription factor 6 (ATF6) (Manie et al., 2014). Both IRE1 $\alpha$ and PERK are type I transmembrane Ser/Thr protein kinases, and possess similar structures with an $\mathrm{NH}_{2}$-terminal ER luminal domain and a cytosolic kinase domain (Pandey et al., 2019). In addition, IRE1 $\alpha$ has an extra RNase domain with endonuclease activity (Abdullah and Ravanan, 2018). ATF6 is a type II transmembrane protein with a $\mathrm{COOH}$-terminal ER luminal domain and an $\mathrm{NH}_{2}$ terminal cytosolic domain with bZIP transcription factor activity (Pandey et al., 2019).

In the absence of ER stress, all three UPR sensors remain inactive resulting from binding to the $78 \mathrm{kDa}$ glucose-regulated protein 78 (GRP78), also known as binding-immunoglobulin protein (BIP), an abundant ER-resident chaperone encoded by gene HSPA5. When mutant, unfolded, or misfolded proteins accumulate in the ER, they release the inhibitory effect of GRP78 on PERK, IRE1 $\alpha$, and ATF6 activity through their high affinity for GRP78 (Oakes and Papa, 2015). In addition, the unfolded or misfolded proteins also act as active ligands for their activation (Credle et al., 2005; Gardner and Walter, 2011). All three UPR branches have outputs to attempt to alleviate ER stress for cell survival by distinct or partially overlapped mechanisms such as mitigating protein misfolding, reducing protein synthesis, or enhancing protein degradation (Figure 1). However, if the adaptive UPR fails to restore ER homeostasis, UPR signaling undergoes continuous activation to initiate prodeath signals through multiple pathways that eventually trigger intrinsic apoptosis (Hetz and Papa, 2018).

\section{AUTOPHAGY}

Autophagy is a highly conserved self-degradation process that delivers aggregated or misfolded proteins, lipid droplets, glycogens, and damaged organelles to lysosomes for degradation. It plays a fundamental role in the homeostasis of almost all types of cells, tissues, and organs (Levine and Kroemer, 2019). Autophagy has been divided into at least three subtypes: macroautophagy, chaperone-mediated autophagy (CMA), and microautophagy, with each involving different mechanisms of substrate delivery to the lysosome. Microautophagy degrades the cytosolic contents through small invaginations in the lysosomal membrane, while CMA mediates selective cytoplasmic proteins with a consensus KFERQ sequence motif into the lysosomal lumen for degradation upon recognition by heat shock cognate $71 \mathrm{kDa}$ protein (HSC70) and targeting by lysosomal associated membrane protein 2A (LAMP2A) (Tekirdag and Cuervo, 2018). Macroautophagy (hereafter referred to autophagy) is the major and most thoroughly characterized autophagic pathway. It 


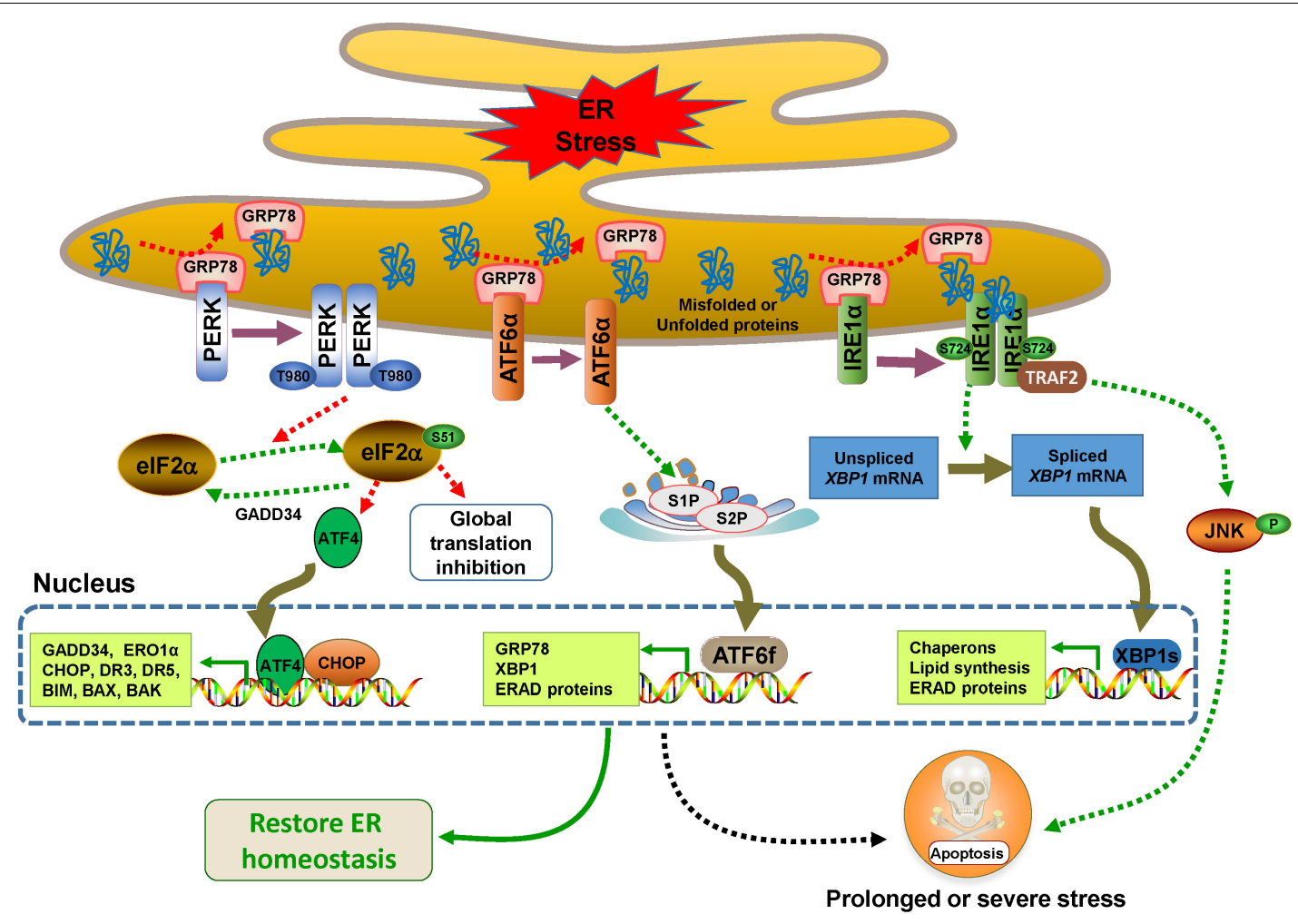

FIGURE 1 | Endoplasmic reticulum (ER) stress and UPR signaling pathways. The UPR is controlled by three major branches, IRE1 $\alpha$, PERK, and ATF6, which bind to GRP78 in the ER under normal conditions. In response to ER stress, the three sensors are activated by dissociating with GPR78. PERK undergoes dimerization, autophosphorylation, and then decreases protein synthesis by phosphorylating elF2 $\alpha$ at the Ser51, which selectively results in the translation of transcription factor 4 (ATF4), a factor that activates transcription of its downstream UPR genes such as CCAAT/enhancer binding protein (C/EBP) homologous protein (CHOP) (Harding et al., 2000). CHOP and ATF4 can upregulate the expression of genes involved in the UPR and apoptosis such as BIM, BAX, BAK, death receptor 3 (DR3), DR5, inositol 1,4,5-trisphosphate (IP3) receptor 1 (IP3R1) and ER oxidase $1 \alpha$ (ERO1 $\alpha$ ) (Iurlaro and Munoz-Pinedo, 2016). When the ER stress is relieved, CHOP and ATF4 induce the expression of growth arrest and DNA damage inducible protein 34 (GADD34), which directly dephosphorylates elF2 $\alpha$ and restarts global mRNA transcription (Novoa et al., 2001). IRE1 $\alpha$ also undergoes dimerization, and even oligomerization and autophosphorylation, and then, its RNase activity is activated and XBP1 mRNA is cleaved to generate XBP1s. The transcription factor XBP1s is responsible for the expression of a subset of downstream genes involved in ERAD, lipid synthesis, protein folding, translocation, and secretion (Korennykh and Walter, 2012; Hetz and Papa, 2018). Activated IRE1 $\alpha$ also interacts with tumor necrosis factor receptor (TNFR)-associated factor-2 (TRAF2) and promotes a cascade of phosphorylation events that ultimately activates Jun amino-terminal kinase (JNK)-mediated cell death (Urano et al., 2000). The dissociation of GRP78 drives ATF6 to translocate to the Golgi, where it is cleaved by site-1 protease (S1P) and S2P to generate ATF6f, which transcriptionally activates the expression of a variety of genes involved in ERAD and ER chaperones including GRP78 and XBP1 (Haze et al., 1999; Shen et al., 2002; Yamamoto et al., 2007; Hillary and FitzGerald, 2018).

sequesters cytoplasmic contents within a double-membrane structure followed by fusion with lysosomes for degradation. According to the type of substrates it degrades, autophagy is also classified into non-selective autophagy and selective autophagy (Shimizu, 2018). Selective autophagy mediates specific substrates or organelles such as aggregate-prone proteins (aggrephagy), mitochondria (mitophagy), peroxisomes (pexophagy), pathogens (xenophagy), and ER (ERphagy) to the autophagic machinery for degradation via adaptor molecules (Stolz et al., 2014).

Under normal conditions, basal autophagy is ongoing at lower levels so that cells will optimally function after the removal of damaged organelles or unwanted substrates (Deegan et al., 2013). Autophagy is dramatically triggered by various stimuli such as nutrient deprivation, accumulation of protein aggregates, oxidative stress, hypoxia, and toxic molecule treatment (Corona Velazquez and Jackson, 2018). The complete processes of autophagy contain multiple sequential steps (autophagy induction, phagophore nucleation, elongation and closure, autophagosome maturation, and fusion with lysosomes to form autolysosomes) with complex mechanisms mediated by autophagy-related gene (ATG) proteins, their partners, and a variety of kinases (Figure 2).

\section{INTERPLAY OF ER STRESS AND AUTOPHAGY}

Although ER stress and autophagy can act as independent drivers, they share many common features such as protecting tissues by alleviating stress and inducing cell death when alleviating failure caused by extreme or chronic stress. Since it was first described in yeast in 2006, a large number of studies have revealed that ER stress and autophagy are mechanistically related (Bernales et al., 2006; Yorimitsu et al., 2006; Rashid et al., 2015). Changing 


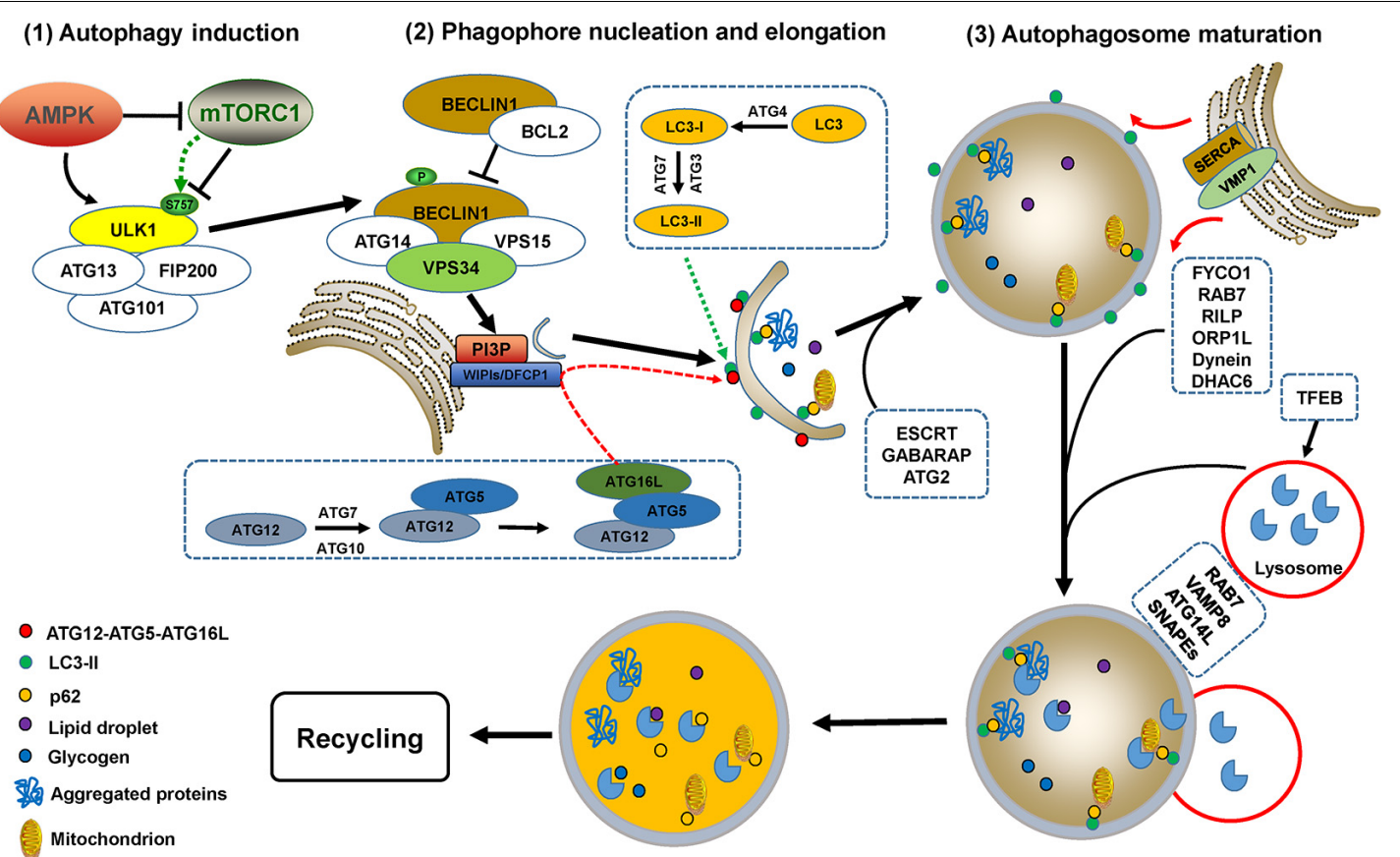

(5) Degradation $\quad$ (4) Autolysosome formation

FIGURE 2 | The processes of autophagy. Autophagy processes include autophagy induction, phagophore nucleation, elongation and closure, autophagosome maturation, and fusion with lysosomes to form autolysosomes, which are mediated by multiple ATG proteins and a variety of kinases or partners with complex mechanisms. Autophagy initiation requires the activation of the uncoordinated-51-like kinase (ULK) complex, which is regulated by two kinases, AMP-activated protein kinase (AMPK) and mammalian target of rapamycin (mTOR) complex 1 (mTORC1). ULK1 activation causes phosphorylation of BECLIN1 and ATG14, thus promoting the formation of the PI3KC3 complex, which enhances the activity of VPS34 to generate phosphatidylinositol 3-phosphate (PI3P) (Russell et al., 2013; Nascimbeni et al., 2017). The enrichment of PI3P recruits double FYVE domain-containing protein 1 (DFCP1), downstream ATG proteins, and WD repeat proteins interacting with phosphoinositides 1 and 2 (WIPI1/2) for phagophore nucleation (Young and Wang, 2018). Phagophore elongation is mediated by two ubiquitin-like conjugation systems, ATG12-ATG5-ATG16L and the ATG8 (light chain 3, LC3) conjugation system (Noda and Inagaki, 2015). Phagophore closure is most likely accomplished by endosomal sorting complex required for transport (ESCRT) machinery-mediated membrane abscission (Tsuboyama et al., 2016; Takahashi et al., 2018, 2019). Additionally, the $\gamma$-aminobutyric acid receptor-associated protein (GABARAP) and ATG2 family are also critical for phagophore closure (Weidberg et al., 2010; Bozic et al., 2020). The ER-localized transmembrane protein vacuole membrane protein 1 (VMP1) mediates ER-phagophore dissociation via activating ER $\mathrm{Ca}^{2+}$ channel sarcoplasmic $\mathrm{Ca}^{2+}$-ATPase (SERCA) and perturbing the local $\mathrm{Ca}^{2+}$ concentration (Zhao et al., 2017). Mature autophagosomes migrate to lysosomes involves the participation of proteins such as RAB7, FYVE coiled-coil domain-containing protein 1 (FYCO1), RAB-interacting lysosomal protein (RILP), Oxysterol-binding protein-related protein 1L (ORP1L), histone deacetylase 6 (HDAC6), kinesin or dynein (Nakamura and Yoshimori, 2017). RAB GTPases, soluble $\mathrm{N}$-ethylmaleimide-sensitive factor attachment protein receptors (SNARES), PI3K complex, and multiple tethering factors are involved in the fusion of autophagosomes with lysosomes to form autolysosomes, in which the autophagosomal contents are degraded by lysosomal acid hydrolases (Martini-Stoica et al., 2016; Zhao and Zhang, 2019). Transcription factor EB (TFEB) is a major regulator for autophagosome formation, lysosomal biogenesis, and lysosomal function (Martini-Stoica et al., 2016; Cortes and La Spada, 2019).

the functions of one system can affect the homeostasis of the other system, in which activation of autophagy triggered by ER stress-induced UPR is dominant. All three UPR branches can directly activate autophagy induction and autophagosome formation during ER stress through activating the expression of multiple ATG genes, inhibiting the expression of several autophagy inhibitors, or modulating several kinases including AMPK or mTORC1 (Figure 3). ER stress also regulates autophagy through mediating $\mathrm{Ca}^{2+}$ release. For example, ER stress stimulates $\mathrm{Ca}^{2+}$ release from the ER lumen into the cytosol through the IP3R channel, which activates the CaMKK $\beta / A M P K$ pathway and inhibits mTORC1 activity, thus triggering autophagy induction. In addition, the presence of $\mathrm{Ca}^{2+}$ in the cytosol leads to death-associated protein kinase 1 (DAPK1) activation, which phosphorylates BCL2 and BECLIN1 to induce autophagy (Verfaillie et al., 2010). However, whether ER stress affects autophagosome maturation, autolysosome formation, or lysosomal degradation is largely unknown.

\section{PERK and Autophagy}

Activation of the PERK pathway contributes to expression of multiple ATG genes (Cai et al., 2016). Under hypoxia, PERKmediated ATF4 and CHOP activation transcriptionally activates LC3 and ATG5 expression (Rouschop et al., 2010; Rzymski et al., 2010). ATG12 mRNA as well as its protein levels are increased by PERK-mediated eIF2 $\alpha$ phosphorylation (Kouroku et al., 2007). ATF4 is sufficient for transcription of several ATG genes including ATG3, BECLIN1, LC3, ATG12, and ATG16L1, whereas CHOP expression transcriptionally upregulates ATG5, ATG10, and GABARAP expression (B'Chir et al., 2013). ATF4/CHOP heterodimer induces the transcriptional expression of $A T G 7$, as well as $p 62$ and neighbor of BRCA1 gene 1 (NBR1), which act as 


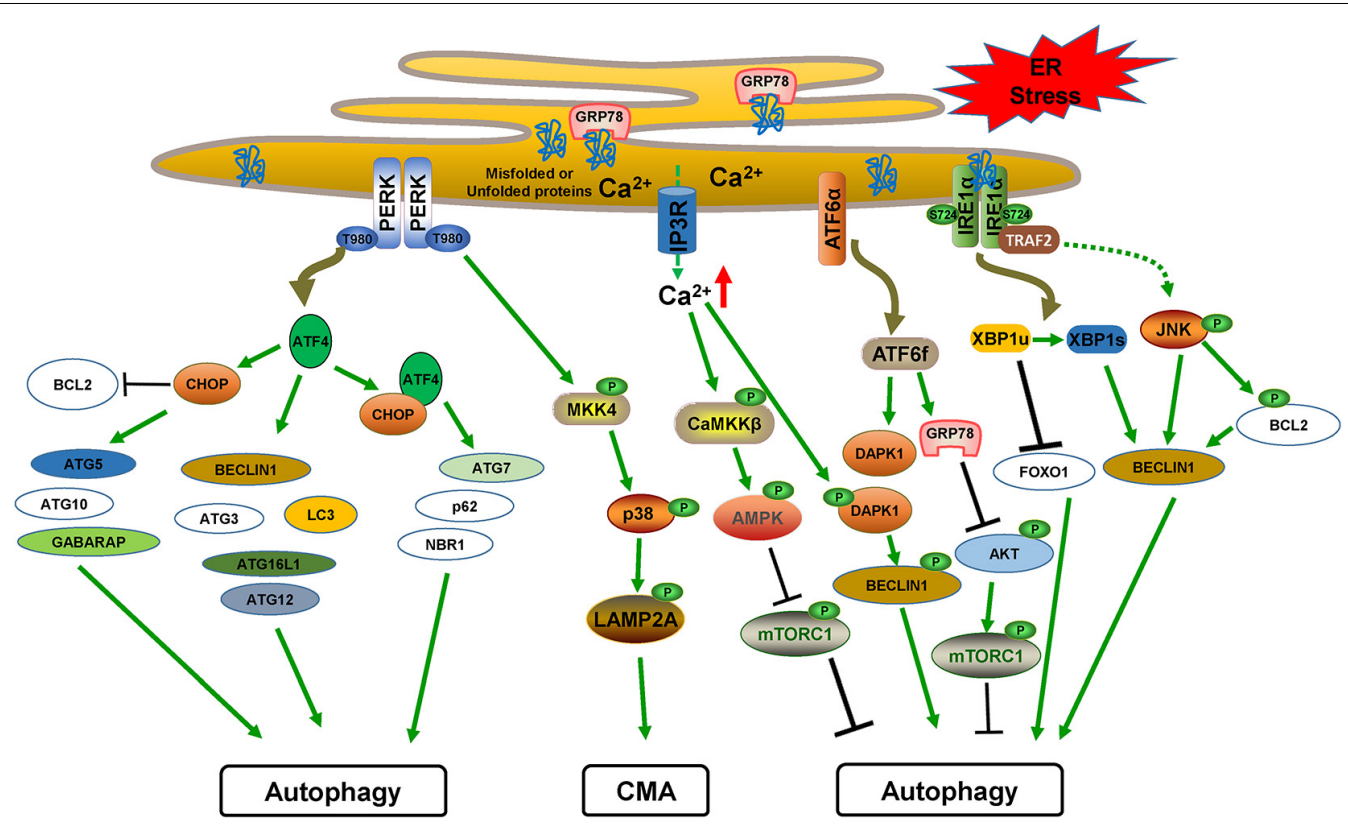

FIGURE 3 | The cross-links between ER stress/UPR and autophagy. PERK-mediated ATF4 translation induces CHOP. The transcriptional factors ATF4 and CHOP induce multiple ATG genes and autophagy regulatory factors alone or together. PERK also activates the MKK4/p38 pathway for CMA activation by phosphorylating LAMP2A. ATF6f activates the expression of GRP78, which inhibits AKT/mTORC1 activation, and DAPK1, which phosphorylates BECLIN1 to induce autophagy. IRE1 $\alpha$ activation leads to JNK signaling, which induces BECLIN1 expression and also phosphorylates BCL2 to dissociate BECLIN1 for autophagy activation. XBP1s generation by IRE1 $\alpha$ activation also transcriptionally activates BECLIN1 expression.

cargo receptors for selective autophagy of ubiquitinated targets (Lamark et al., 2009; B'Chir et al., 2013). CHOP can also promote autophagy through inhibiting the expression of BCL2, a protein that sequesters BECLIN1 in the ER and inhibits autophagosome formation (Pattingre et al., 2005; Rzymski et al., 2010).

The PERK pathway also initiates autophagy by activating AMPK and inhibiting mTORC1 activity (Avivar-Valderas et al., 2013). In addition, PERK-mediated ATF4/CHOP expression induces autophagy by inhibiting mTORC1 activity (Salazar et al., 2009; Bruning et al., 2013). Recently, The TFEBmediated GADD34 expression may integrate mTORC1-mediated autophagy and ER stress (Gambardella et al., 2020). ER stress can also activate the CMA pathway by PERK activation, which recruits mitogen-activated protein kinase 4 (MKK4) and activates p38 to phosphorylate LAMP2A for CMA activation (Li et al., 2017).

\section{IRE1 $\alpha$ and Autophagy}

IRE1 $\alpha$ activation phosphorylates c-Jun N-terminal kinase (JNK) (Ogata et al., 2006), and this activation induces autophagy by directly phosphorylating BCL2 and disrupting its interaction with BECLIN1, which induces autophagosome formation (Wei et al., 2008). Moreover, JNK activation also upregulates BECLIN1 transcription (Li et al., 2009; Ren et al., 2010). The IRE1dependent activation of AMPK is also involved in autophagy initiation (Meares et al., 2011). The generation of XBP1s, which is dependent on IRE1 $\alpha$ RNase activity, also induces autophagy through direct transcriptional activation of BECLIN1 expression (Margariti et al., 2013). Interestingly, the unspliced form of XBP1
(XBP1u) negatively regulates autophagy through interacting with forkhead box O1 (FOXO1) and decreasing its levels (Zhou et al., 2011; Vidal et al., 2012; Zhao et al., 2013), suggesting that IRE1 $\alpha$-mediated XBP1 splicing during ER stress is critical for autophagy induction.

\section{ATF6 and Autophagy}

It has been suggested that the activation of the ATF6 branch triggers autophagy. ATF6-mediated GRP78 transcriptional expression induces autophagy initiation by inhibiting AKT activity (Yung et al., 2011). In addition, during ER stress, formed ATF6 $f$ induces the expression of DAPK1 (Kalvakolanu and Gade, 2012; Gade et al., 2014), which phosphorylates BECLIN1 so that it subsequently dissociates from its negative regulator BCL2, thus promoting autophagosome formation (Zalckvar et al., 2009).

\section{ER STRESS, UPR, AND AUTOPHAGY CROSS TALK IN PD PATHOGENESIS}

Dopaminergic neurons are particularly sensitive to unfolded, misfolded, and excessively aggregated proteins. ER stress and autophagy impairment are two essential events that lead to the imbalance of proteostasis, which contributes to DA neurodegeneration. In recent years, a large number of studies have focused on the relationship between ER stress and PD pathogenesis. For example, injection of an ER stress inducer, tunicamycin, into mouse brains causes high levels of oligomeric $\alpha$-SYN, DA neuron death, locomotor deficiency, and 


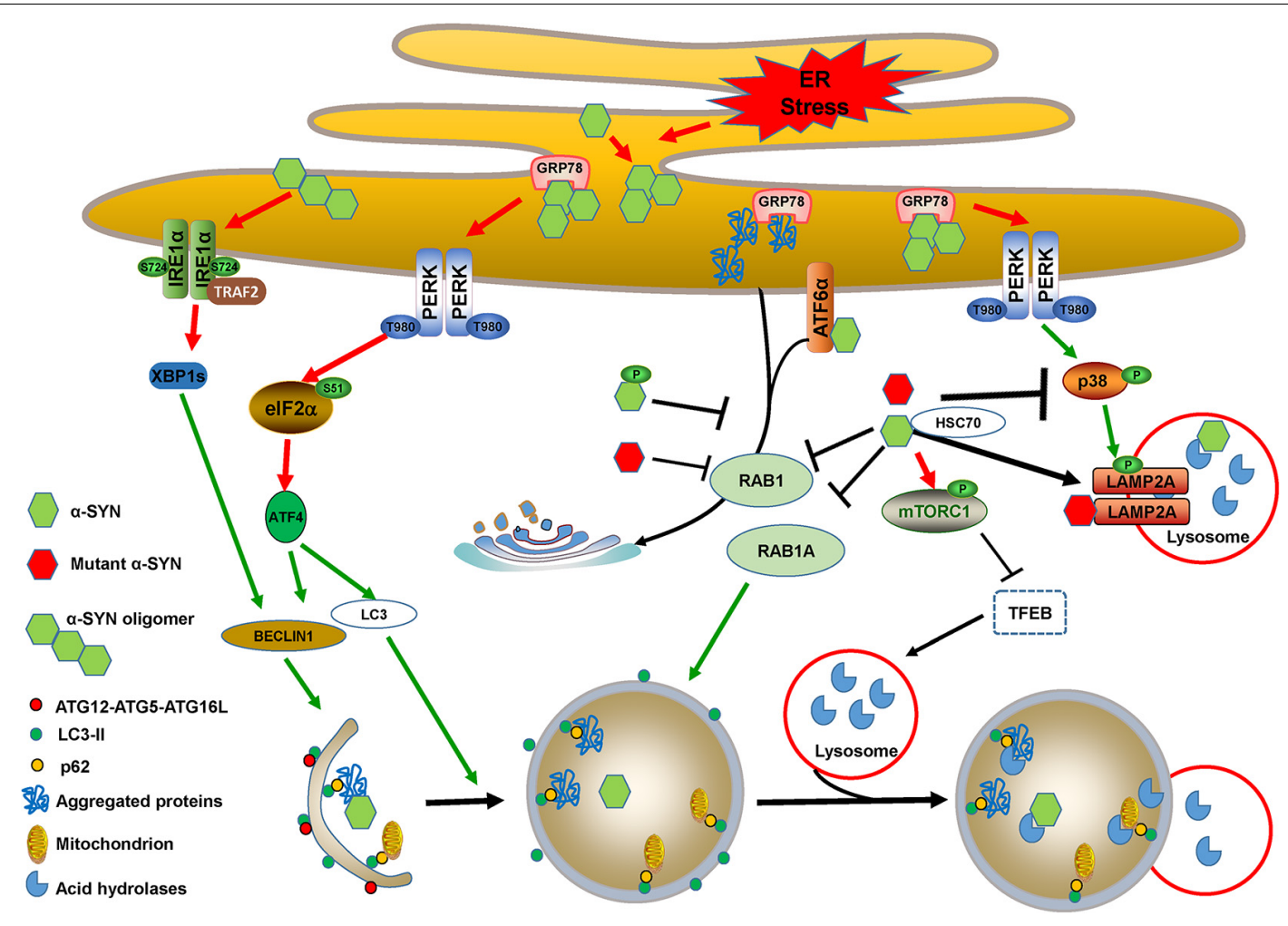

FIGURE 4 | Cross-links between ER stress and autophagy in $\alpha$-SYN-mediated pathology. Accumulated $\alpha$-SYN binds to GRP78 and activates ER stress. $\alpha$-SYN also induces ER stress by binding to ATF6 and inhibiting its translocation to Golgi bodies. Additionally, wild-type, mutant, or phosphorylated $\alpha$-SYN activates ER stress by inhibiting ER-Golgi trafficking, which leads to the accumulation of aggregated proteins. PERK/ATF4 activation that occurred due to ER stress induces BECLIN1 and LC3 expression. Oligomeric $\alpha$-SYN directly activates the IRE1 $\alpha /$ XBP1s branch and thus induces BECLIN1 expression. The expression of BECLIN1 and LC3 triggers autophagy induction. However, wild-type and mutant $\alpha$-SYN inhibit autophagosome maturation by repressing RAB1A function. They also activate mTORC1 and sequester TFEB in the cytoplasm to block autophagic flux by impairing lysosomal biogenesis and function. PERK activation-mediated MKK4/p38/LAMP2A phosphorylation may promote CMA for wild-type $\alpha$-SYN degradation, whereas wild-type and mutant $\alpha$-SYN directly inhibit p38 activation to block CMA, and mutant $\alpha$-SYN can also inhibit CMA uptake by interacting with LAMP2A.

glial activation (Coppola-Segovia et al., 2017), suggesting that administration of an ER stress inducer into the SNpc could be a novel animal model of PD. However, impaired autophagy, CMA, and mitophagy are frequently observed rather than autophagy activation in PD. Interestingly, conditional deletion of the Atg7 gene in mice recapitulates many of the pathologic features of $\mathrm{PD}$, including age-related loss of DA neurons, loss of striatal DA, accumulation of $\alpha-S Y N$, and ubiquitinated protein aggregates (Ahmed et al., 2012). Thus far, it is generally recognized that in PD patients as well as various PD cellular and animal models, ER stress activation, UPR markers, and autophagy dysfunction undoubtedly exist in the lesion regions, and are closely related to both genetic and neurotoxic factors that induce DA neurodegeneration.

\section{ER Stress, UPR Activation, and Autophagy Dysfunction in Tissues of PD Patients}

The UPR activation markers, including phosphorylation of PERK, eIF2 $\alpha$, and IRE1 $\alpha$, are observed in neuromelanincontaining DA neurons in the postmortem $\mathrm{SNpc}$ of $\mathrm{PD}$ patients rather than age-matched controls (Hoozemans et al., 2007; Hoozemans et al., 2012; Heman-Ackah et al., 2017). In addition, the immunoreactivity of phosphorylated PERK is colocalized with increased $\alpha$-SYN immunoreactivity in DA neurons (Hoozemans et al., 2007). Importantly, UPR activation is an early event in neurodegeneration and is closely associated with the accumulation and aggregation of $\alpha$-SYN (Hoozemans et al., 2012). GRP78 and CHOP, which are ER stress markers, are increased in the SNpc in PD patients (Selvaraj et al., 2012). Moreover, GRP78 is increased to a greater extent in dementia with LB (DLB) and PD with dementia (PDD) patients in the cingulate gyrus and parietal cortex (Baek et al., 2016). GRP78 is also dramatically upregulated in the brain tissues of autosomal recessive juvenile $\mathrm{PD}$ (AR-JP) patients caused by a loss of functional Parkin, a familial PD genetic factor (Imai et al., 2001). This is due to the defective function of Parkin's E3 ligase activity, which results in a lack of degradation and subsequent accumulation of its substrate, the PAEL receptor.

The protein disulfide isomerase (PDI) family participates in disulfide bond formation, reduction, isomerization, and accurate folding of nascent proteins, and they also are increased to constitute an adaptive response to ER stress 
(Turano et al., 2002). It was found that a PDI member, pancreatic PDI (PDIp), accumulates in PD patient tissues (Conn et al., 2004). Homocysteine-induced ER protein (HERP) is a stress response protein that functions in ER folding and ERADmediated degradation, and ER load reduction is also increased in the SN of PD patients (Slodzinski et al., 2009). However, all PD patients do not experience an increase in the UPR in tissues. Baek et al. (2019) found that GRP78 mRNA levels are upregulated, but they also showed that the protein levels of GRP78 are decreased in several brain regions, including the cingulate gyrus in $\mathrm{PD}$ patients. Recently, similar findings suggested that both GRP78 and ATF4 protein levels are decreased in the SNpc in PD patients (Esteves and Cardoso, 2020). These inconsistent results may be related to the different pathological degrees of PD patients. In the early stage of PD, UPR activation is an adaptive response to protect DA neurons from damage. However, in the late stage, excessive stress-induced neuron damage or severe DA neuron loss leads to inhibition of expression of these ER stress markers. Finally, ER stress and UPR activation are undoubted pathological processes in PD.

It was first discovered that autophagic degeneration of DA neurons occurred in the $\mathrm{SN}$ regions of PD patients in early studies (Anglade et al., 1997). Later, immunopositivity for LC3II indicated that autophagosome formation occurred in the majority of LBs and LNs, and LC3-II colocalized with $\alpha$-SYN in PD patients (Alvarez-Erviti et al., 2010; Dehay et al., 2010; Tanji et al., 2011), while the amount of lysosomes, and levels and activities of glucocerebrosidase (GCase) or protease cathepsin D (CTSD) were decreased within DA neurons in PD tissues (Dehay et al., 2010; Moors et al., 2019). Moreover, enlarged mitochondria have been observed within autophagosomes using confocal laser scanning microscopy in PD brains, suggesting PD-associated abnormal mitophagy (Zhu et al., 2003). Fiesel and Springer (2015); Fiesel et al. (2015) and Hou et al. (2018) confirmed that mitophagy indicated by ubiquitin Ser65 phosphorylation is specifically increased in PD patients and correlates with levels of LBs. Levels of HSC70 and LAMP2A were also dramatically decreased, which indicated that the CMA activity is significantly reduced in the SN tissues of PD patients (Alvarez-Erviti et al., 2010; Murphy et al., 2015).

The selective loss of LAMP2A protein and decreased levels of HSC70 were directly correlated with the increase in $\alpha$-SYN levels and the accumulation of cytosolic CMA substrate proteins in PD samples (Murphy et al., 2015). Interestingly, crowded organelles and lipid membranes, including dystrophic lysosomes, mitochondria, and autophagosome-like structures, were observed in a recent PD postmortem study (Shahmoradian et al., 2019). Together, impairments in autophagy and CMA have been found in lesion regions in PD patients compared with matched controls using postmortem tissues. The increased autophagosomes and failed lysosomal clearance are common hallmarks in SN tissues of PD patients. The activated ER stress and UPR pathway may affect increases in autophagosome formation through the mechanisms described above. However, whether and how ER stress/UPR affects autophagosome fusion with lysosomes or lysosomal functions in $\mathrm{PD}$ are largely unclear.

\section{Association of PD Genetic Factors With ER Stress, UPR, and Autophagy}

Several PD-related genetic factors including gain of function of $\alpha$-SYN and LRRK2, and loss of function of Parkin, PINK1, and DJ-1, affect ER stress/UPR and autophagy, and discussing the roles and mechanisms of these genetic factors in these two biological processes can greatly promote the understanding of $\mathrm{PD}$ pathology.

\section{$\alpha-S Y N$ in ER Stress, UPR, and Autophagy}

$\alpha$-SYN is encoded by the SNCA gene, the mutations of which such as A53T, or duplication or triplication, have been linked to autosomal-dominant forms of PD. Aggregated $\alpha$-SYN, especially the accumulation in the brain of its soluble oligomers, is one of most important causative factors for both hereditary and sporadic PD. $\alpha-S Y N$ is a major component deposited in LBs and LNs, in which it harbors extensive phosphorylation at Ser129, which mediates its aggregation and toxicity (Wang et al., 2020a). $\alpha$-SYN can be degraded via multiple clearance machineries, including autophagy, CMA, and the ubiquitin proteasome system (Hou et al., 2020). Although wild-type, mutant, phosphorylated, and oligomeric $\alpha$-SYN activate ER stress and promote autophagy induction, they block autophagic flux by impairing autophagosome maturation, fusion with lysosomes, and lysosomal biogenesis or functions (Figure 4).

$\alpha-S Y N$ overexpression and its aggregated neurotoxic forms activate all three UPR branches and trigger chronic ER stressinduced apoptosis. It was discovered that overexpression of both wild-type and A53T mutant $\alpha$-SYN affects RAB1, which is involved in trafficking substrates from the ER to the Golgi bodies, thus inducing UPR activation by blocking ER-Golgi trafficking. RAB1 overexpression reduces stress and protects against DA neurodegeneration in PD animal models (Cooper et al., 2006), and the Ser129 phosphorylation of $\alpha$-SYN may mediate the ER-Golgi traffic disruption and trigger PERK/eIF2 $\alpha$ branch activation in in vitro PD models (Sugeno et al., 2008; Jiang et al., 2010). A30P $\alpha$-SYN disrupts the Golgi morphology and facilitates the susceptibility to ER stress. A53T $\alpha$-SYN upregulates GRP78 levels and eIF2 $\alpha$ phosphorylation, and results in mitochondrial cell death in neurons, as well as in astrocytes (Smith et al., 2005; Liu et al., 2018; Paiva et al., 2018).

Accumulation of $\alpha$-SYN within the ER activates the PERK pathway by directly interacting with GRP78 in vitro and in vivo (Bellucci et al., 2011). $\alpha$-SYN oligomers rather than monomers also activate the IRE1 $\alpha$-XBP1 pathway (CastilloCarranza et al., 2012). In addition, $\alpha$-SYN reduces ATF6 processing and leads to ERAD impairment by directly binding to ATF6 or indirectly restricting its incorporation into coat protein complex II (COPII) vesicles (Credle et al., 2015). ER stress also leads to the accumulation of $\alpha$-SYN oligomers (Jiang et al., 2010), suggesting that ER stress plays an important role in $\alpha$-SYN neurotoxicity. It is interesting to hypothesize whether $\alpha$-SYN toxicity is a cause or a consequence of ER stress and UPR dysfunction. Additional evidence indicates that $\alpha$-SYN accumulation within the ER is required for UPR activation, and toxic $\alpha$-SYN oligomer formation precedes ER stress and UPR 
activation (Colla et al., 2012a,b). Nevertheless, collaboration of $\alpha$-SYN accumulation-induced ER stress and ER stress-enhanced $\alpha$-SYN neurotoxicity is vital for PD pathogenesis.

Both wild-type and A53T mutant $\alpha$-SYN can promote autophagy induction by upregulating BECLIN1 and LC3 expression (Yu et al., 2009; Decressac et al., 2013). The upregulation of BECLIN1 and LC3 expression may be involved in $\alpha$-SYN-mediated ER stress and activation of the PERK/eIF2 $\alpha /$ ATF4 pathway. However, wild-type $\alpha-S Y N$ overexpression impairs autophagic flux via RAB1A inhibition, leads to ATG9 mislocalization, and inhibits the formation of autophagosomes (Winslow et al., 2010). RAB1 overexpression protects DA neurodegeneration in various PD animal models (Cooper et al., 2006; Gitler et al., 2008; Coune et al., 2012). Additionally, $\alpha$-SYN binds to high mobility group box 1 (HMGB1) and strengthens BECLIN-BCL2 binding by blocking HMGB1-BECLIN1 interaction (Song et al., 2014). Both wildtype and A53T $\alpha$-SYN can induce mTOR activity and impair autophagy (Jiang et al., 2013; Gao et al., 2015). In an AAVmediated $\alpha$-SYN overexpression mouse model, $\alpha$-SYN impairs autophagic efflux by sequestering TFEB in the cytoplasm, accompanied by p62 and LC3-II accumulation (Decressac et al., 2013). Similarly, PC12 cells harboring A53T $\alpha$-SYN display accumulated autophagic-vesicular structures and impaired lysosomal hydrolysis (Stefanis et al., 2001). Primary neurons seeded with $\alpha$-SYN fibrils exist in phosphorylated $\alpha$-SYN species with high neurotoxicity, named " $\mathrm{p} \alpha-S Y N^{*}$." These result from incomplete autophagic degradation of $\alpha-S Y N$, which colocalizes with GRP78 at mitochondria-associated ER membranes (Grassi et al., 2018), and this may play a role in the cross-linking between ER stress, mitochondrial fission, and mitophagy.

Interestingly, wild-type $\alpha$-SYN contains a KFERQ sequence and is greatly degraded through the CMA pathway, while pathogenic $\alpha$-SYN mutants act as CMA uptake inhibitors through interacting with LAMP2A on the lysosomal membrane (Cuervo et al., 2004). AAV-mediated overexpression of A53T $\alpha$-SYN in neurons or A53T $\alpha$-SYN in transgenic mice results in a reduction of CMA-mediated proteolysis of other substrates (Xilouri et al., 2009; Malkus and Ischiropoulos, 2012). Interestingly, modification of wild-type $\alpha$-SYN by DA also impairs CMA proteolysis, similar to mutant $\alpha$-SYN (MartinezVicente et al., 2008), which enables us to understand the selective vulnerability of the SNpc in PD. Although PERK activation has an active effect on LAMP2A through activating MKK4/p38 (Li et al., 2017), PERK activation induced by $\alpha-S Y N$ is insufficient for activating LAMP2A activity, as both wildtype and pathogenic $\alpha$-SYN have been reported that can directly inhibit p38 activation (Iwata et al., 2001). Whether wild-type and pathogenic $\alpha$-SYN have an effect on phosphorylation and the activity of LAMP2A is not clear.

\section{Parkin and PINK1 in ER Stress, UPR, and Autophagy}

Mutations in Parkin (Kitada et al., 1998) and PTEN inducible kinase 1 (PINK1) (Valente et al., 2004a) have been identified as the most common causes of autosomal-recessive early-onset PD. Parkin mutations account for nearly $50 \%$ of young PD patients, and PINK1 mutations account for 1-9\% (Lucking et al., 2000;
Puschmann, 2013). Parkin is an E3 ligase and functions in the ERAD of misfolded ER proteins (Shimura et al., 2000; Senft and Ronai, 2015). Parkin is upregulated by ATF4 under either ER stress or mitochondrial stress and inhibits stressinduced mitochondrial dysfunction and cell death via its E3 ligase activity (Imai et al., 2000; Bouman et al., 2011). Conversely, an accumulation of PAEL receptor, a substrate of Parkin, induces ER stress and cell death (Imai et al., 2001). Furthermore, the ER stress inhibitor salubrinal prevents rotenone-induced ER stress and cell death through the ATF4-Parkin pathway (Wu et al., 2014).

Parkin also regulates ER stress and UPR via transcription factor p53-dependent XBP1 transcription regulation (Duplan et al., 2013). It has been reported that Parkin participates in ER stress regulation in DA neurons and also in astrocytes (Ledesma et al., 2002; Singh et al., 2018). Moreover, an activation of the PERK branch of the UPR was observed that was induced by defective mitochondria, and PERK inhibition is neuroprotective in parkin mutant flies (Celardo et al., 2016), as well as in flies harboring mutants of pink1, a gene in which mutations in humans are associated with both genetic and sporadic PD (Valente et al., 2004a,b). It has been shown that PINK1 inhibits ER stress-induced damage to mouse primary cortical neurons ( $\mathrm{Li}$ and $\mathrm{Hu}, 2015)$, and downregulation of ER stress response genes has been detected in aged Pink1 knockout mice (Torres-Odio et al., 2017).

Parkin and PINK1 are two important regulators that control mitophagy and mitochondrial homeostasis (Swerdlow and Wilkins, 2020). Mitophagy, the process of removing damaged mitochondria, is compromised in PD pathogenesis, and its dysfunction is closely associated with DA neurodegeneration (Liu et al., 2019). The ATF4/CHOP heterodimer transcriptionally activates expression of p62 and NBR1 (B'Chir et al., 2013), which are two cargo adaptors involved in Parkin/PINK1-mediated mitophagy, and may be underlying the role of ER stress in mitophagy (Nguyen et al., 2016). It has been reported that the ER stress induced by tunicamycin (TM) and thapsigargin (TG) prevents Parkin loss and promotes its recruitment to the mitochondria, and also activates mitophagy during reperfusion after ischemia (Zhang et al., 2014). However, the crosslink and mechanisms between ER stress and mitophagy in PD pathogenesis are mainly unknown.

\section{LRRK2 in ER Stress, UPR, and Autophagy}

Mutations in the leucine-rich repeat kinase 2 (LRKK2) gene are the most common cause of autosomal-dominant forms of $\mathrm{PD}$, as well as more than $3 \%$ of sporadic PD cases (Costa et al., 2020; Tolosa et al., 2020). LRRK2 possesses kinase function for catalyzing substrates and GTPase function for GTPGDP hydrolysis. LRRK2 is co-localized with ER markers in DA neurons (Vitte et al., 2010), and LRKK2 depletion results in GRP78 downregulation in response to 6-hydroxydopamine (6-OHDA)-induced ER stress (Yuan et al., 2011). LRRK2 phosphorylates leucyl-tRNA synthetase (LRS), and this increases the number of misfolded proteins, causes ER stress, and induces autophagy initiation (Ho et al., 2018). LRRK2-G2019S mutation exacerbates these processes. It has recently been reported that LRRK2 regulates ER-mitochondria tethering through the 
PERK-mediated activation of E3 ligases, and LRKK2 mutation enhances the sensitivity to ER stress and decreases mitochondrial biogenesis (Toyofuku et al., 2020). In addition, mutant LRKK2 binds to SERCA to repress its activity, leading to ER $\mathrm{Ca}^{2+}$ depletion and triggering ER stress, which ultimately results in mitochondrial $\mathrm{Ca}^{2+}$ overload and mitochondrial dysfunction in astrocytes (Lee et al., 2019).

It has been reported that LRRK2 functions in endosomaland vesicle-trafficking pathways, plays roles in cytoskeleton dynamics and neurite outgrowth, and regulates multiple steps of the autophagy-lysosome pathway (Madureira et al., 2020). Although wild-type and mutant LRRK2 promote autophagy by ER stress induced by the phosphorylation of LRS, they impair the autophagic degradation in an LRS-independent manner (Ho et al., 2018). LRKK2-G2019S fibroblasts exhibit higher autophagic activity levels involved in activating ERK activity rather than the mTOR pathway (Bravo-San Pedro et al., 2013). An early and transient phosphorylation of ERK1/2 is involved in ER stress activation (Arai et al., 2004), indicating that LRKK2 may regulate autophagy initiation through the ERK pathway via LRS-meditated ER stress. LRRK2 and LRRK2G2019S also regulate p62 phosphorylation, influence its affinity to ubiquitinated cargo (Park et al., 2016; Kalogeropulou et al., 2018), and decrease autophagic protein degradation. Additionally, LRKK2 and LRKK2-G2019S inhibit autophagosome formation and autophagosome-lysosome fusion in various LRRK2-related PD models may through regulating phosphorylation of a number of RAB proteins (Madureira et al., 2020). LRKK2 pathogenic mutants also impair lysosomal function, which is detected by abnormal lysosomal morphology, abnormal cellular lysosomal localization, increased lysosomal pH, or inhibition of lysosomal enzymes (Madureira et al., 2020).

Like $\alpha$-SYN, LRKK2 can also be degraded by CMA, and unlike $\alpha$-SYN mutants, which increase the binding affinity of HSC70 and LAMP2A, LRRK2 mutants block the formation of the CMA translocation complex by inducing LAMP2A and HSC70 accumulation at the lysosomal membrane (Orenstein et al., 2013; Ho et al., 2020). Although it has been reported that LRRK2G2019S binds to MKK4/7 (Zhu et al., 2013), it is unclear whether LRRK2-G2019S participates in the PERK/MKK4/p38 pathway in CMA. Together, the promotion of autophagy initiation by LRRK2 and its pathogenic mutants is partly due to ER stress and UPR activation. However, they most likely inhibit autophagic flux, as well as CMA and lysosomal functions in an ER stressindependent manner.

\section{DJ-1 in ER Stress, UPR, and Autophagy}

DJ-1, a protein encoded by the PARK7 gene, assumes multiple functions including antioxidative stress and chaperone properties (Mencke et al., 2021). Mutations or deletions of DJ-1 are associated with autosomal-recessive early-onset forms of PD (Bonifati et al., 2003). DJ-1 regulates ER stress and UPR by binding to and stabilizing ATF4 mRNA under both basal and stress conditions (Yang et al., 2019). DJ-1 can also protect against ER stress-induced cell death in Neuro2a cells (Yokota et al., 2003). Moreover, it has been shown that oxidized DJ-1 can interact with N-terminal arginylated GPR78, and thus facilitate the self-polymerization of p62 and the targeting of p62-cargo complexes to phagophores under oxidative stress (Lee et al., 2018). Interestingly, DJ-1 expression is regulated under ER stress such that XBP1 directly binds to its promoter and stimulates its expression (Duplan et al., 2013).

Overexpression of DJ-1 in DA neurons and in the SN of rat brains promotes ERK-dependent autophagy. Although there are no obvious effects of DJ-1 deficiency on autophagy in SHSY5Y cells (Gonzalez-Polo et al., 2009; Ren et al., 2010), the loss of DJ-1 perturbs paraquat-induced autophagic initiation in SH-SY5Y cells by enhancing the mTOR activity (Gonzalez-Polo et al., 2009). In addition, DJ-1 deficiency in microglia impairs autophagy-mediated p62 degradation and reduces microglialmediated $\alpha$-SYN phagocytosis (Nash et al., 2017). DJ-1 protects against DA neurodegeneration through enhancing CMA in PD animal models, SH-SY5Y cells, and astrocytes (Xu et al., 2017; De Miranda et al., 2018). Together, the upregulation of DJ-1 expression in response to ER stress may enhance the CMA or autophagic degradation of aggregated proteins, which bridges the close link between ER stress and autophagy in PD pathogenesis.

\section{Association of PD Neurotoxins With ER Stress and UPR}

The well-known parkinsonian inducers including 1-methyl-4-phenyl-1,2,3,6-tetrahydropyridine (MPTP)/1methyl-4-phenylpyridinium $\left(\mathrm{MPP}^{+}\right), 6-\mathrm{OHDA}$, and rotenone, all have profound effects on the regulation of ER stress/UPR and autophagy. Discussing the cross-link between ER stress/UPR and autophagy in these parkinsonian inducers may promote our understanding of PD pathology.

\section{MPTP/MPP+ in ER Stress, UPR, and Autophagy}

MPTP is the best-known chemical for inducing a PD model in vivo. Intraperitoneally injecting mice with MPTP reproduces PD pathology, including the selective loss of DA neurons in the SN and accumulation of protein aggregates, and eventually leads to the onset of PD-like clinical symptoms (Bove et al., 2005). MPTP can efficiently cross the blood-brain barrier and is metabolized to the destructive $\mathrm{MPP}^{+}$by glial monoamine oxidase $\mathrm{B}$ (MAO-B). $\mathrm{MPP}^{+}$is transported into DA neurons via dopamine transporter (DAT) and induces DA neuron death by inhibiting mitochondrial functions and increasing mitochondrial superoxide.

$\mathrm{MPP}^{+}$triggers a significant increase in the expression of UPR genes including ATF4 and CHOP, which are involved in the activation of IRE $1 \alpha$ and PERK in various cellular models (Ryu et al., 2002; Holtz and O'Malley, 2003). $\mathrm{MPP}^{+}$has been shown to activate cyclin-dependent-like kinase 5 (CDK5)-mediated $\mathrm{XBP} 1 s$ phosphorylation, which favors its nuclear translocation and promotes its transcriptional activity in rat primary cultured neurons (Jiao et al., 2017). The intracerebral injection of $\mathrm{MPP}^{+}$ into the SNpc of rabbit brains induces ER stress involving the activation of the ATF6 and NF- $\mathrm{B}$ signaling pathways (Ghribi et al., 2003). MPTP also promotes the phosphorylation of p38 and enhances the interaction between phosphorylated p38 and ATF6, leading to an increase in ATF6 transcriptional activity (Egawa et al., 2011). $\mathrm{MPP}^{+}$also activates the expression of UPR markers 
such as PDIp, which accumulates in PD patient tissues (Conn et al., 2004). MPTP/MPP ${ }^{+}$activate ER stress and expression of the UPR markers GRP78 and CHOP by disturbing ER $\mathrm{Ca}^{2+}$ levels, which is accompanied by a decrease in AKT/mTOR activity (Selvaraj et al., 2012), and is closely associated with autophagy activation.

The regulatory effects of $\mathrm{MPTP} / \mathrm{MPP}^{+}$on autophagy are not consistent among different studies. MPTP/MPP ${ }^{+}$treatment increases autophagosome formation, increases p62 levels, and decreases lysosomal activity (Dehay et al., 2010; Lim et al., 2011; Lim et al., 2014; Jovanovic-Tucovic et al., 2019), indicating that autophagy initiation is activated but autophagic flux is blocked by $\mathrm{MPP}^{+}$treatment. The activation of autophagy initiation by $\mathrm{MPP}^{+}$treatment probably occurs through activation of AMPK (Jovanovic-Tucovic et al., 2019). Further study indicates that mild $\mathrm{MPP}^{+}$exposure (10 or $200 \mu \mathrm{M}$ for $48 \mathrm{~h}$ ) predominantly inhibits autophagic degradation by reducing lysosomal hydrolase cathepsin $\mathrm{D}$ activity, whereas acute $\mathrm{MPP}^{+}$treatment $(2.5$ and $5 \mathrm{mM}$ for $24 \mathrm{~h}$ ) inhibits both autophagic degradation and basal autophagy by decreasing lysosomal density (Miyara et al., 2016). $\mathrm{MPP}^{+}$treatment increases autophagy activation and promotes mitochondrial degradation, which involves the activation of ERK signaling rather than the canonical pathway (Zhu et al., 2007, 2012; Dagda et al., 2008).

We speculate that $\mathrm{MPP}^{+}$treatment-induced mitochondrial degradation may not be due to the activation of autophagic flux, but it dramatically disrupts mitochondrial functions and impairs mitochondrial biogenesis (Zhu et al., 2012). $\mathrm{MPP}^{+}$ treatment-induced ER stress and the UPR closely contribute to autophagy induction and autophagic flux blockage. All branches of the UPR are activated by MPTP/MPP ${ }^{+}$treatment, as well as subsequent events such as activation of AMPK (JovanovicTucovic et al., 2019), decreased mTOR activity (Selvaraj et al., 2012), and increased NF- $\mathrm{B}$ signaling (Ghribi et al., 2003),

TABLE 1 | The roles and mechanisms of PD-related factors in ER stress, autophagy and their cross-links.

\begin{tabular}{|c|c|c|c|}
\hline PD-related factors & Roles and mechanisms in ER stress & Roles and mechanisms in autophagy & Cross-links \\
\hline$\alpha-S Y N$ & $\begin{array}{l}\alpha \text {-SYN directly binds to GRP78 to activate } \\
\text { PERK and } \alpha \text {-SYN reduces ATF6 processing; } \\
\alpha-S Y N \text { oligomers activate the IRE1 } \alpha \text {-XBP1 } \\
\text { pathway; Wild-type and mutant } \alpha-S Y N \text { affect } \\
\text { RAB1 and disrupts traffic from the ER to the } \\
\text { Golgi; ER stress leads to the accumulation of } \\
\alpha-S Y N \text { oligomers. }\end{array}$ & $\begin{array}{l}\alpha \text {-SYN promotes autophagy induction by } \\
\text { upregulating BECLIN1 and LC3 expression; } \\
\alpha \text {-SYN impairs autophagic flux via RAB1A } \\
\text { inhibition, TFEB sequestration and lysosomal } \\
\text { inhibition; } \alpha \text {-SYN is degraded via autophagy and } \\
\text { CMA; Oxidized } \alpha \text {-SYN and A53T } \alpha \text {-SYN reduce } \\
\text { CMA-mediated proteolysis through binding to } \\
\text { LAMP2A or inhibit p38 activation. }\end{array}$ & $\begin{array}{l}\alpha \text {-SYN-mediated BECLIN1 and LC3 } \\
\text { expression via ER stress activation may } \\
\text { be involved in its role in autophagy } \\
\text { induction; The inhibition of RAB } \\
\text { pathway by } \alpha \text {-SYN is both involved in } \\
\text { ER stress activation and autophagic } \\
\text { flux impairment. }\end{array}$ \\
\hline Parkin/PINK1 & $\begin{array}{l}\text { Parkin is upregulated by ER stress; Parkin } \\
\text { inhibits PERK-mediated ER stress through its } \\
\text { E3 ligase activity; Parkin also regulates ER } \\
\text { stress via p53-XBP1 pathway; PINK1 inhibits } \\
\text { ER stress via PERK branch. }\end{array}$ & $\begin{array}{l}\text { Parkin and PINK1 control mitochondrial } \\
\text { homeostasis by enhancing mitophagy. }\end{array}$ & $\begin{array}{l}\text { PERK/ATF4/CHOP induced Parkin, p62 } \\
\text { and NBR expression may be involved in } \\
\text { Parkin/PINK1-mediated mitophagy. }\end{array}$ \\
\hline LRRK2 & $\begin{array}{l}\text { LRRK2 is partly localized in ER; LRRK2 and } \\
\text { its pathogenic mutant G2019S phosphorylate } \\
\text { LRS, and cause ER stress; Mutant LRKK2 } \\
\text { binds to SERCA and leads to ER } \mathrm{Ca}^{2+} \\
\text { depletion to trigger ER stress; }\end{array}$ & $\begin{array}{l}\text { LRRK2 and LRKK2-G2019S induce autophagy } \\
\text { initiation by phosphorylating LRS; } \\
\text { LRRK2-G2019S promotes autophagy by } \\
\text { activating ERK; LRRK2 and LRRK2-G2019S } \\
\text { decrease autophagic degradation by regulating } \\
\text { p62 phosphorylation, as well as inhibit } \\
\text { autophagosome and autolysosome formation via } \\
\text { phosphorylating a number of RAB proteins. }\end{array}$ & $\begin{array}{l}\text { LRKK2 and it mutants trigger } \\
\text { autophagy initiation by activating ERK } \\
\text { via LRS-meditated accumulation of } \\
\text { misfolded proteins and ER stress. } \\
\text { LRKK2 and it mutants activate ER } \\
\text { stress and impair autophagic flux may } \\
\text { also through regulating RABs functions. }\end{array}$ \\
\hline DJ-1 & $\begin{array}{l}\text { DJ-1 regulates ER stress/UPR by binding to } \\
\text { and stabilizing ATF4 mRNA; Oxidized DJ-1 } \\
\text { interact with arginylated GPR78; DJ-1 is } \\
\text { upregulated under ER stress through XBP1 } \\
\text { branch. }\end{array}$ & $\begin{array}{l}\text { DJ-1 promotes ERK-dependent autophagy; Loss } \\
\text { of DJ-1 perturbs paraquat-induced autophagic } \\
\text { initiation by enhancing the mTOR activity; DJ-1 } \\
\text { deficiency in microglia impairs } \\
\text { autophagy-mediated p62 degradation and } \\
\text { reduces microglial-mediated } \alpha \text {-SYN } \\
\text { phagocytosis. DJ-1 enhances CMA activity }\end{array}$ & $\begin{array}{l}\text { Oxidized DJ-1 interact with arginylated } \\
\text { GPR78 and facilitate p62-cargo } \\
\text { complexes to phagophore; ER stress } \\
\text { induced DJ-1 upregulation enhances } \\
\text { the CMA or autophagic degradation. }\end{array}$ \\
\hline MPTP/MPP ${ }^{+}$ & $\begin{array}{l}\text { MPTP/MPP }+ \text { activates IRE1 } \alpha, \text { PERK, and } \\
\text { ATF6 branches through enhancing CDK5 and } \\
\text { p38 activity, as well as disturbing ER } \mathrm{Ca}^{2+} \\
\text { levels. }\end{array}$ & $\begin{array}{l}\text { MPTP/MPP+ treatment increases autophagy } \\
\text { initiation but blocks autophagic flux, probably } \\
\text { through activating AMPK and ERK activity, and } \\
\text { reducing mTOR activity and lysosomal hydrolase } \\
\text { activity. }\end{array}$ & $\begin{array}{l}\text { MPTP/MPP+ treatment-induced ER } \\
\text { stress and the UPR activation } \\
\text { contribute to autophagy induction. }\end{array}$ \\
\hline 6-OHDA & $\begin{array}{l}\text { 6-OHDA activates ER stress/UPR by } \\
\text { phosphorylating PERK and elF2 } \alpha \text {. }\end{array}$ & $\begin{array}{l}\text { 6-OHDA treatment elicits autophagy activation by } \\
\text { activating ERK and AMPK activity, as well as } \\
\text { BECLIN1 expression; 6-OHDA promotes } \\
\text { autophagic flux and CMA activity. }\end{array}$ & $\begin{array}{l}\text { 6-OHDA-activated ER stress/UPR } \\
\text { contributes to excessive autophagy } \\
\text { initiation and autophagic flux. }\end{array}$ \\
\hline Rotenone & $\begin{array}{l}\text { Rotenone triggers ER stress involving } \\
\text { activation of all three branches of PERK, } \\
\text { IRE1 } \alpha \text {, and ATF6. }\end{array}$ & $\begin{array}{l}\text { Rotenone treatment increases autophagy } \\
\text { induction but inhibits autophagic flux by impairing } \\
\text { Iysosomal functions; rotenone increases } \\
\text { mitophagy. }\end{array}$ & $\begin{array}{l}\text { Rotenone-mediated ER stress/UPR } \\
\text { stimulates autophagy induction. }\end{array}$ \\
\hline
\end{tabular}


which contribute to autophagy initiation (Zhu et al., 2017). MPTP/MPP ${ }^{+}$treatment also disrupts lysosomal functions and leads to autophagic flux blockage (Dehay et al., 2010; Lim et al., 2011). The cross-link between autophagy initiation activation and autophagic flux blockage exaggerates the damage to DA neurons by MPTP/MPP ${ }^{+}$treatment.

\section{6-OHDA in ER Stress, UPR, and Autophagy}

6-OHDA, a selective catecholaminergic neurotoxin, is also widely used to induce DA neuron death as a PD model by eliciting the production of mitochondrial and cytosolic reactive oxygen species (ROS) (Simola et al., 2007). Increased levels of GRP78 and CHOP expression, as well as phosphorylated PERK and eIF $2 \alpha$, are detected in DA cellular models that are subjected to 6-OHDA treatment (Ryu et al., 2002; Holtz and O’Malley, 2003; Yamamuro et al., 2006; Deng et al., 2012; Xie et al., 2012; Ning et al., 2019b).
Like $\mathrm{MPP}^{+}$, 6-OHDA treatment also elicits autophagy activation and promotes mitochondrial degradation involving the activation of ERK signaling (Dagda et al., 2008). Unlike $\mathrm{MPP}^{+}$, 6-OHDA induces AMPK phosphorylation, followed by mTOR dephosphorylation, and increases LC3 conversion, p62 degradation, and cytoplasmatic acidification in SH-SY5Y cells (Arsikin et al., 2012). A recent study indicated that 6-OHDA treatment induces excessive autophagy with increased AMPK activity, decreased mTOR activity, reduced p62 levels, and also prevents alterations in lysosomal functions (Chung et al., 2018). In addition, 6-OHDA treatment stimulates CMA activity by increasing LAMP2A levels (Wang et al., 2018). 6-OHDA treatment induces BECLIN1 and decreases BCL2 expression and p62 levels, which are inhibited by PERK inhibition (Ning et al., 2019a,b), and this directly cross-links the ER stress and autophagy in 6-OHDA-induced PD pathogenesis. Together,

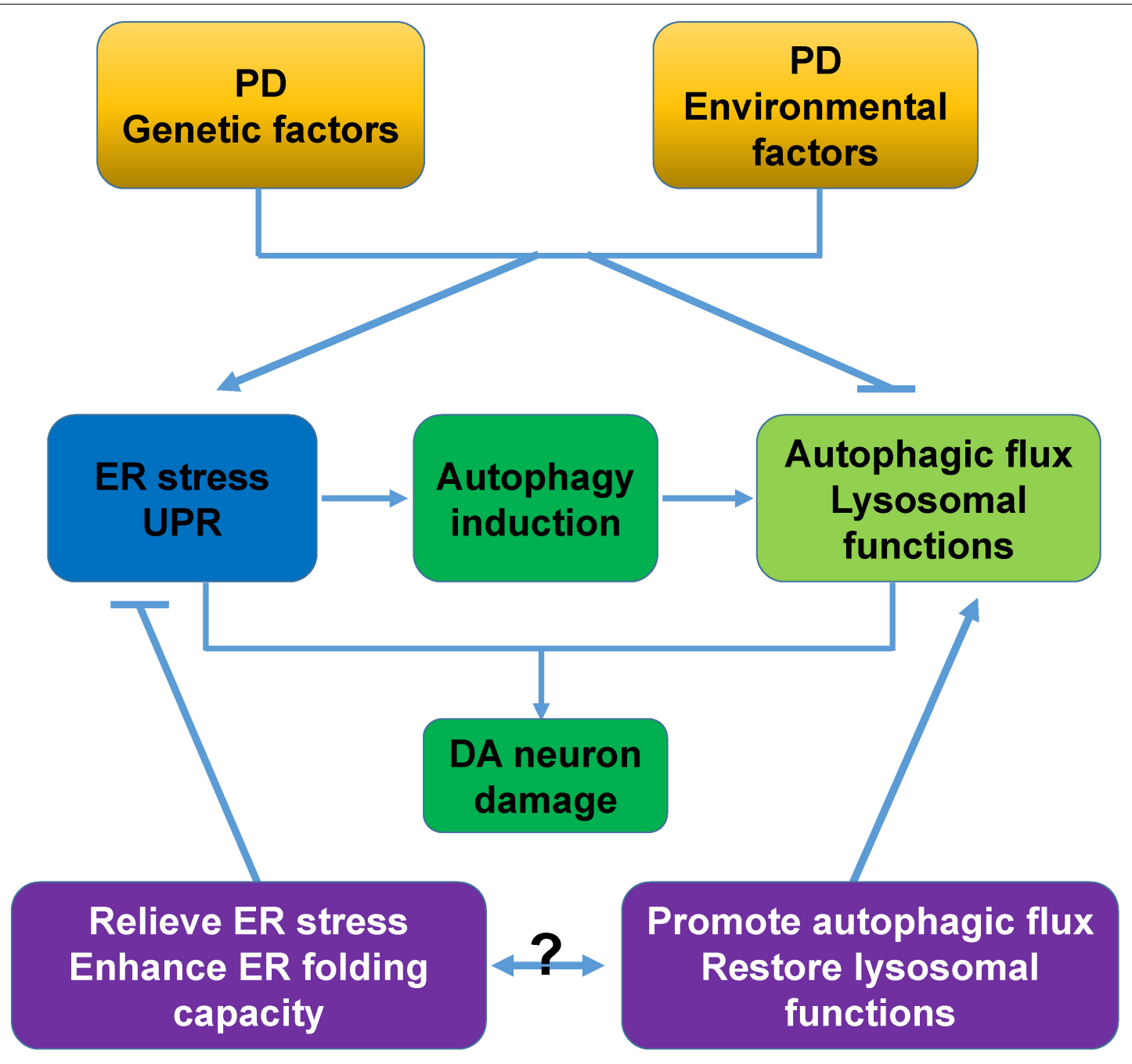

FIGURE 5 | Proposed model of cross-links between ER stress, autophagy, and DA neurodegeneration. PD-associated genetic and environmental factors trigger ER stress, and ER stress activates the UPR and induces autophagy to alleviate cellular stress. However, these PD-associated factors commonly block autophagic flux and impair lysosomal functions, and these changes synergistically cause severe damage and degeneration of DA neurons. Relieving ER stress/UPR, enhancing the ER folding capacity, promoting autophagic flux, and restoring lysosomal functions are neuroprotective for PD. The combined intervention of ER stress/UPR and autophagy would be a more attractive therapeutic approach for PD. 
6-OHDA-induced excessive autophagy activation and autophagic flux contribute to $\mathrm{PD}$ pathogenesis.

\section{Rotenone in ER Stress, UPR, and Autophagy}

Rotenone treatment produces most of the movement disorder symptoms and the histopathological features of $\mathrm{PD}$, including LBs (Betarbet et al., 2002). Rotenone also triggers ATF4 and CHOP expression involving activation of IRE1 $\alpha$ and PERK in cellular models (Ryu et al., 2002; Ramalingam et al., 2019). An induction of the IRE $1 \alpha$ and PERK branch of the UPR has also been shown in rotenone rat or mouse models of PD (Tong et al., 2016a,b; Ramalingam et al., 2019). Treatment of N2a cells with rotenone triggers ER stress and the UPR involving all three branches of PERK, IRE1 $\alpha$, and ATF6 (Gupta et al., 2019).

Rotenone induces an increase in autophagy related proteins LC3-II and BECLIN1, as well as in autophagy substrates such as $\alpha$-SYN and p62 in cultured PC12 cells (Wu et al., 2015) and in SH-SY5Y cells (Xiong et al., 2013), suggesting that rotenone exerts bidirectional effects on autophagy initiation and autophagic flux. Rotenone increases oligomeric wild-type and A53T $\alpha$-SYN in transfected cells through inhibiting their autophagic degradation (Yu et al., 2009). Similar to $\mathrm{MPP}^{+}$, although rotenone treatment results in decreased autophagic flux, it increases mitophagy for mitochondrial degradation (Giordano et al., 2014), suggesting that the mitochondrialspecific degradation pathway used by $\mathrm{MPP}^{+}$and rotenone may be independent from that of autophagy. Therefore, rotenoneinduced ER stress and the UPR initiate autophagy induction but block autophagic flux by impairing lysosomal functions, which aggravates the imbalance of cellular homeostasis and damage to DA neurons.

\section{FUTURE PERSPECTIVES}

The accumulation of unfolded, misfolded, and aggregated proteins, and the accumulation induced by cellular stress are essential mechanisms underlying the causes of PD. ER stress/UPR and autophagy, two major pathways that are used to respond to proteostasis imbalance, play especially important roles in $\mathrm{PD}$ pathology. In this review, we systematically examined the intrinsic molecular links between ER stress, the UPR, and autophagy, as well as the roles of these cross-links in PD pathology. ER stress, UPR activation, and dysregulated autophagy commonly coexist in patients and various cellular and animal models of $\mathrm{PD}$, and are closely related to $\mathrm{DA}$ neurodegeneration caused by PD genetic and neurotoxic factors (Table 1). This is why targeting one of these processes would create a beneficial PD treatment (Moors et al., 2017; Martinez et al., 2019). More importantly, combining both ER stress/UPR and autophagy regulation, such as relieving ER stress/UPR or enhancing the ER folding capacity, and

\section{REFERENCES}

Abdullah, A., and Ravanan, P. (2018). The unknown face of IRE1alpha - beyond ER stress. Eur. J. Cell Biol. 97, 359-368. doi: 10.1016/j.ejcb.2018.05.002 promoting autophagic flux or restoring lysosomal functions will be more neuroprotective for PD (Figure 5). In addition, the ER stress/UPR activation, upregulation of UPR genes, and accumulation of autophagosome markers and autophagic substrates are expected to be useful biomarkers or diagnostic parameters of PD.

For example, administration of GSK2606414, a PERK inhibitor, results in effective neuroprotection and prevents loss of SNpc DA neurons in mice that were treated with PD neurotoxin 6-OHDA (Mercado et al., 2018). Gene therapy that restores the folding capacity by administration of viralmediated overexpression of GRP78 (Gorbatyuk et al., 2012) or UPR transcriptional factor XBP1s (Sado et al., 2009; Valdes et al., 2014) in the $\mathrm{SNpc}$ results in neuroprotection against neurotoxin- or genetic factors-induced damage to DA neurons. Rapamycin, an inhibitor of mTOR, initiates autophagy induction, enhances autophagic flux (Rubinsztein and Nixon, 2010), and confers significant protective effects on DA neurons in various PD models (Moors et al., 2017). Additionally, enhancing lysosomal biogenesis by TFEB overexpression or pharmacological stimulation of TFEB function by CCI-779 was shown to eliminate $\alpha$-SYN oligomers and rescue midbrain DA neurons from $\alpha$-SYN toxicity in rats (Decressac et al., 2013).

It is notable that PD-associated genetic or environmental factors lead to ER stress and UPR activation, which commonly initiate autophagy. However, these PD-associated factors also block autophagic flux and impair lysosomal functions. An intervention strategy for one of the two processes alone may not completely alleviate the imbalance in cellular homeostasis. However, a combined treatment strategy for both ER stress/UPR and the autophagy pathway has not yet been studied in PD. We recognize that combining ER stress/UPR and autophagy to explore the pathological mechanisms of $\mathrm{PD}$ and develop interventional strategies that combine ER stress/UPR and autophagy will be very meaningful in the future.

\section{AUTHOR CONTRIBUTIONS}

GW and XL designed the theme of the manuscript. HR and $\mathrm{WZ}$ wrote the manuscript. All authors approved the submitted version.

\section{FUNDING}

This research was funded by the National Natural Science Foundation of China (31970966, 32070970, and 31871023), Suzhou Clinical Research Center of Neurological Disease (Szzx201503), and the Priority Academic Program Development of Jiangsu Higher Education Institutions.
Ahmed, I., Liang, Y., Schools, S., Dawson, V. L., Dawson, T. M., and Savitt, J. M. (2012). Development and characterization of a new Parkinson's disease model resulting from impaired autophagy. J. Neurosci. 32, 16503-16509. doi: 10.1523/ JNEUROSCI.0209-12.2012 
Alvarez-Erviti, L., Rodriguez-Oroz, M. C., Cooper, J. M., Caballero, C., Ferrer, I., Obeso, J. A., et al. (2010). Chaperone-mediated autophagy markers in Parkinson disease brains. Arch. Neurol. 67, 1464-1472. doi: 10.1001/archneurol. 2010.198

Anglade, P., Vyas, S., Javoy-Agid, F., Herrero, M. T., Michel, P. P., Marquez, J., et al. (1997). Apoptosis and autophagy in nigral neurons of patients with Parkinson's disease. Histol. Histopathol. 12, 25-31.

Arai, K., Lee, S. R., van Leyen, K., Kurose, H., and Lo, E. H. (2004). Involvement of ERK MAP kinase in endoplasmic reticulum stress in SH-SY5Y human neuroblastoma cells. J. Neurochem. 89, 232-239. doi: 10.1111/j.1471-4159.2004. 02317.x

Arsikin, K., Kravic-Stevovic, T., Jovanovic, M., Ristic, B., Tovilovic, G., Zogovic, N., et al. (2012). Autophagy-dependent and -independent involvement of AMP-activated protein kinase in 6-hydroxydopamine toxicity to SH-SY5Y neuroblastoma cells. Biochim. Biophys. Acta 1822, 1826-1836. doi: 10.1016/j. bbadis.2012.08.006

Avivar-Valderas, A., Bobrovnikova-Marjon, E., Alan Diehl, J., Bardeesy, N., Debnath, J., and Aguirre-Ghiso, J. A. (2013). Regulation of autophagy during ECM detachment is linked to a selective inhibition of mTORC1 by PERK. Oncogene 32, 4932-4940. doi: 10.1038/onc.2012.512

Baek, J. H., Mamula, D., Tingstam, B., Pereira, M., He, Y., and Svenningsson, P. (2019). GRP78 level is altered in the brain, but not in plasma or cerebrospinal fluid in Parkinson's disease patients. Front. Neurosci. 13:697. doi: 10.3389/fnins. 2019.00697

Baek, J. H., Whitfield, D., Howlett, D., Francis, P., Bereczki, E., Ballard, C., et al. (2016). Unfolded protein response is activated in Lewy body dementias. Neuropathol. Appl. Neurobiol. 42, 352-365. doi: 10.1111/nan.12260

B'Chir, W., Maurin, A. C., Carraro, V., Averous, J., Jousse, C., Muranishi, Y., et al. (2013). The eIF2alpha/ATF4 pathway is essential for stress-induced autophagy gene expression. Nucleic Acids Res. 41, 7683-7699. doi: 10.1093/nar/gkt563

Bellucci, A., Navarria, L., Zaltieri, M., Falarti, E., Bodei, S., Sigala, S., et al. (2011). Induction of the unfolded protein response by alpha-synuclein in experimental models of Parkinson's disease. J. Neurochem. 116, 588-605. doi: 10.1111/j.14714159.2010.07143.x

Bernales, S., McDonald, K. L., and Walter, P. (2006). Autophagy counterbalances endoplasmic reticulum expansion during the unfolded protein response. PLoS Biol. 4:e423. doi: 10.1371/journal.pbio.0040423

Betarbet, R., Sherer, T. B., and Greenamyre, J. T. (2002). Animal models of Parkinson's disease. Bioessays 24, 308-318. doi: 10.1002/bies.10067

Bonifati, V., Rizzu, P., van Baren, M. J., Schaap, O., Breedveld, G. J., Krieger, E., et al. (2003). Mutations in the DJ-1 gene associated with autosomal recessive early-onset parkinsonism. Science 299, 256-259. doi: 10.1126/science.1077209

Bouman, L., Schlierf, A., Lutz, A. K., Shan, J., Deinlein, A., Kast, J., et al. (2011). Parkin is transcriptionally regulated by ATF4: evidence for an interconnection between mitochondrial stress and ER stress. Cell Death Differ. 18, 769-782. doi: $10.1038 /$ cdd. 2010.142

Bove, J., Prou, D., Perier, C., and Przedborski, S. (2005). Toxin-induced models of Parkinson's disease. NeuroRx 2, 484-494. doi: 10.1602/neurorx.2.3.484

Bozic, M., van den Bekerom, L., Milne, B. A., Goodman, N., Roberston, L., Prescott, A. R., et al. (2020). A conserved ATG2-GABARAP family interaction is critical for phagophore formation. EMBO Rep. 21:e48412. doi: 10.15252/embr. 201948412

Bravo-San Pedro, J. M., Niso-Santano, M., Gomez-Sanchez, R., Pizarro-Estrella, E., Aiastui-Pujana, A., Gorostidi, A., et al. (2013). The LRRK2 G2019S mutant exacerbates basal autophagy through activation of the MEK/ERK pathway. Cell. Mol. Life Sci. 70, 121-136. doi: 10.1007/s00018-012-1061-y

Bruning, A., Rahmeh, M., and Friese, K. (2013). Nelfinavir and bortezomib inhibit mTOR activity via ATF4-mediated sestrin-2 regulation. Mol. Oncol. 7, 10121018. doi: 10.1016/j.molonc.2013.07.010

Cai, Y., Arikkath, J., Yang, L., Guo, M. L., Periyasamy, P., and Buch, S. (2016). Interplay of endoplasmic reticulum stress and autophagy in neurodegenerative disorders. Autophagy 12, 225-244. doi: 10.1080/15548627.2015.1121360

Castillo-Carranza, D. L., Zhang, Y., Guerrero-Munoz, M. J., Kayed, R., RinconLimas, D. E., and Fernandez-Funez, P. (2012). Differential activation of the ER stress factor XBP1 by oligomeric assemblies. Neurochem. Res. 37, 1707-1717. doi: 10.1007/s11064-012-0780-7

Celardo, I., Costa, A. C., Lehmann, S., Jones, C., Wood, N., Mencacci, N. E., et al. (2016). Mitofusin-mediated ER stress triggers neurodegeneration in pink1/parkin models of Parkinson's disease. Cell Death Dis. 7:e2271. doi: 10. 1038/cddis.2016.173

Chung, Y., Lee, J., Jung, S., Lee, Y., Cho, J. W., and Oh, Y. J. (2018). Dysregulated autophagy contributes to caspase-dependent neuronal apoptosis. Cell Death Dis. 9:1189. doi: 10.1038/s41419-018-1229-y

Colla, E., Coune, P., Liu, Y., Pletnikova, O., Troncoso, J. C., Iwatsubo, T., et al. (2012a). Endoplasmic reticulum stress is important for the manifestations of alpha-synucleinopathy in vivo. J. Neurosci. 32, 3306-3320. doi: 10.1523/ JNEUROSCI.5367-11.2012

Colla, E., Jensen, P. H., Pletnikova, O., Troncoso, J. C., Glabe, C., and Lee, M. K. (2012b). Accumulation of toxic alpha-synuclein oligomer within endoplasmic reticulum occurs in alpha-synucleinopathy in vivo. J. Neurosci. 32, 3301-3305. doi: 10.1523/JNEUROSCI.5368-11.2012

Conn, K. J., Gao, W., McKee, A., Lan, M. S., Ullman, M. D., Eisenhauer, P. B., et al. (2004). Identification of the protein disulfide isomerase family member PDIp in experimental Parkinson's disease and Lewy body pathology. Brain Res. 1022, 164-172. doi: 10.1016/j.brainres.2004.07.026

Cooper, A. A., Gitler, A. D., Cashikar, A., Haynes, C. M., Hill, K. J., Bhullar, B., et al. (2006). Alpha-synuclein blocks ER-Golgi traffic and Rab1 rescues neuron loss in Parkinson's models. Science 313, 324-328. doi: 10.1126/science.1129462

Coppola-Segovia, V., Cavarsan, C., Maia, F. G., Ferraz, A. C., Nakao, L. S., Lima, M. M., et al. (2017). ER stress induced by tunicamycin triggers alpha-synuclein oligomerization, dopaminergic neurons death and locomotor impairment: a new model of Parkinson's disease. Mol. Neurobiol. 54, 5798-5806. doi: 10.1007/ s12035-016-0114-x

Corona Velazquez, A. F., and Jackson, W. T. (2018). So many roads: the multifaceted regulation of autophagy induction. Mol. Cell. Biol. 38:e00303-18. doi: 10.1128/MCB.00303-18

Cortes, C. J., and La Spada, A. R. (2019). TFEB dysregulation as a driver of autophagy dysfunction in neurodegenerative disease: molecular mechanisms, cellular processes, and emerging therapeutic opportunities. Neurobiol. Dis. 122, 83-93. doi: 10.1016/j.nbd.2018.05.012

Costa, C. A. D., Manaa, W. E., Duplan, E., and Checler, F. (2020). The endoplasmic reticulum stress/unfolded protein response and their contributions to Parkinson's disease physiopathology. Cells 9:2495. doi: 10.3390/cells91 12495

Coune, P. G., Schneider, B. L., and Aebischer, P. (2012). Parkinson's disease: gene therapies. Cold Spring Harb. Perspect. Med. 2:a009431. doi: 10.1101/cshperspect. a009431

Credle, J. J., Finer-Moore, J. S., Papa, F. R., Stroud, R. M., and Walter, P. (2005). On the mechanism of sensing unfolded protein in the endoplasmic reticulum. Proc. Natl. Acad. Sci. U.S.A. 102, 18773-18784. doi: 10.1073/pnas.0509487102

Credle, J. J., Forcelli, P. A., Delannoy, M., Oaks, A. W., Permaul, E., Berry, D. L., et al. (2015). alpha-Synuclein-mediated inhibition of ATF6 processing into COPII vesicles disrupts UPR signaling in Parkinson's disease. Neurobiol. Dis. 76, 112-125. doi: 10.1016/j.nbd.2015.02.005

Cuervo, A. M., Stefanis, L., Fredenburg, R., Lansbury, P. T., and Sulzer, D. (2004). Impaired degradation of mutant alpha-synuclein by chaperone-mediated autophagy. Science 305, 1292-1295. doi: 10.1126/science.1101738

Dagda, R. K., Zhu, J., Kulich, S. M., and Chu, C. T. (2008). Mitochondrially localized ERK2 regulates mitophagy and autophagic cell stress: implications for Parkinson's disease. Autophagy 4, 770-782. doi: 10.4161/auto.6458

De Miranda, B. R., Rocha, E. M., Bai, Q., El Ayadi, A., Hinkle, D., Burton, E. A., et al. (2018). Astrocyte-specific DJ-1 overexpression protects against rotenoneinduced neurotoxicity in a rat model of Parkinson's disease. Neurobiol. Dis. 115, 101-114. doi: 10.1016/j.nbd.2018.04.008

Decressac, M., Mattsson, B., Weikop, P., Lundblad, M., Jakobsson, J., and Bjorklund, A. (2013). TFEB-mediated autophagy rescues midbrain dopamine neurons from alpha-synuclein toxicity. Proc. Natl. Acad. Sci. U.S.A. 110, E1817E1826. doi: 10.1073/pnas.1305623110

Deegan, S., Saveljeva, S., Gorman, A. M., and Samali, A. (2013). Stress-induced selfcannibalism: on the regulation of autophagy by endoplasmic reticulum stress. Cell. Mol. Life Sci. 70, 2425-2441. doi: 10.1007/s00018-012-1173-4

Dehay, B., Bove, J., Rodriguez-Muela, N., Perier, C., Recasens, A., Boya, P., et al. (2010). Pathogenic lysosomal depletion in Parkinson's disease. J. Neurosci. 30, 12535-12544. doi: 10.1523/JNEUROSCI.1920-10.2010

Deng, C., Tao, R., Yu, S. Z., and Jin, H. (2012). Inhibition of 6-hydroxydopamineinduced endoplasmic reticulum stress by sulforaphane through the activation 
of Nrf2 nuclear translocation. Mol. Med. Rep. 6, 215-219. doi: 10.3892/mmr. 2012.894

Duplan, E., Giaime, E., Viotti, J., Sevalle, J., Corti, O., Brice, A., et al. (2013). ERstress-associated functional link between Parkin and DJ-1 via a transcriptional cascade involving the tumor suppressor p53 and the spliced X-box binding protein XBP-1. J. Cell Sci. 126(Pt 9), 2124-2133. doi: 10.1242/jcs.127340

Egawa, N., Yamamoto, K., Inoue, H., Hikawa, R., Nishi, K., Mori, K., et al. (2011). The endoplasmic reticulum stress sensor, ATF6alpha, protects against neurotoxin-induced dopaminergic neuronal death. J. Biol. Chem. 286, 79477957. doi: $10.1074 /$ jbc.M110.156430

Esteves, A. R., and Cardoso, S. M. (2020). Differential protein expression in diverse brain areas of Parkinson's and Alzheimer's disease patients. Sci. Rep. 10:13149. doi: 10.1038/s41598-020-70174-z

Fiesel, F. C., Ando, M., Hudec, R., Hill, A. R., Castanedes-Casey, M., Caulfield, T. R., et al. (2015). (Patho-)physiological relevance of PINK1-dependent ubiquitin phosphorylation. EMBO Rep. 16, 1114-1130. doi: 10.15252/embr. 201540514

Fiesel, F. C., and Springer, W. (2015). Disease relevance of phosphorylated ubiquitin (p-S65-Ub). Autophagy 11, 2125-2126. doi: 10.1080/15548627.2015. 1091912

Gade, P., Manjegowda, S. B., Nallar, S. C., Maachani, U. B., Cross, A. S., and Kalvakolanu, D. V. (2014). Regulation of the death-associated protein kinase 1 expression and autophagy via ATF6 requires apoptosis signal-regulating kinase 1. Mol. Cell. Biol. 34, 4033-4048. doi: 10.1128/MCB.00397-14

Gambardella, G., Staiano, L., Moretti, M. N., De Cegli, R., Fagnocchi, L., Di Tullio, G., et al. (2020). GADD34 is a modulator of autophagy during starvation. Sci. $A d v$. 6:eabb0205. doi: 10.1126/sciadv.abb0205

Gao, S., Duan, C., Gao, G., Wang, X., and Yang, H. (2015). Alpha-synuclein overexpression negatively regulates insulin receptor substrate 1 by activating mTORC1/S6K1 signaling. Int. J. Biochem. Cell Biol. 64, 25-33. doi: 10.1016/j. biocel.2015.03.006

Gardner, B. M., and Walter, P. (2011). Unfolded proteins are Ire1-activating ligands that directly induce the unfolded protein response. Science 333, 1891-1894. doi: $10.1126 /$ science. 1209126

Ghemrawi, R., and Khair, M. (2020). Endoplasmic reticulum stress and unfolded protein response in neurodegenerative diseases. Int. J. Mol. Sci. 21:6127. doi: $10.3390 /$ ijms 21176127

Ghribi, O., Herman, M. M., Pramoonjago, P., and Savory, J. (2003). MPP+ induces the endoplasmic reticulum stress response in rabbit brain involving activation of the ATF-6 and NF-kappaB signaling pathways. J. Neuropathol. Exp. Neurol. 62, 1144-1153. doi: 10.1093/jnen/62.11.1144

Giordano, S., Dodson, M., Ravi, S., Redmann, M., Ouyang, X., Darley Usmar, V. M., et al. (2014). Bioenergetic adaptation in response to autophagy regulators during rotenone exposure. J. Neurochem. 131, 625-633. doi: 10.1111/jnc. 12844

Gitler, A. D., Bevis, B. J., Shorter, J., Strathearn, K. E., Hamamichi, S., Su, L. J., et al. (2008). The Parkinson's disease protein alpha-synuclein disrupts cellular Rab homeostasis. Proc. Natl. Acad. Sci. U.S.A. 105, 145-150. doi: 10.1073/pnas. 0710685105

Gonzalez-Polo, R., Niso-Santano, M., Moran, J. M., Ortiz-Ortiz, M. A., Bravo-San Pedro, J. M., Soler, G., et al. (2009). Silencing DJ-1 reveals its contribution in paraquat-induced autophagy. J. Neurochem. 109, 889-898. doi: 10.1111/j.14714159.2009.06020.x

Gorbatyuk, M. S., Shabashvili, A., Chen, W., Meyers, C., Sullivan, L. F., Salganik, M., et al. (2012). Glucose regulated protein 78 diminishes alpha-synuclein neurotoxicity in a rat model of Parkinson disease. Mol. Ther. 20, 1327-1337. doi: $10.1038 / \mathrm{mt} .2012 .28$

Grassi, D., Howard, S., Zhou, M., Diaz-Perez, N., Urban, N. T., Guerrero-Given, D., et al. (2018). Identification of a highly neurotoxic alpha-synuclein species inducing mitochondrial damage and mitophagy in Parkinson's disease. Proc. Natl. Acad. Sci. U.S.A. 115, E2634-E2643. doi: 10.1073/pnas.1713849115

Gupta, S., Biswas, J., Gupta, P., Singh, A., Tiwari, S., Mishra, A., et al. (2019). Salubrinal attenuates nitric oxide mediated PERK:IRE1alpha: ATF-6 signaling and DNA damage in neuronal cells. Neurochem. Int. 131:104581. doi: 10.1016/ j.neuint.2019.104581

Harding, H. P., Novoa, I., Zhang, Y., Zeng, H., Wek, R., Schapira, M., et al. (2000). Regulated translation initiation controls stress-induced gene expression in mammalian cells. Mol. Cell 6, 1099-1108. doi: 10.1016/s1097-2765(00)00108-8
Haze, K., Yoshida, H., Yanagi, H., Yura, T., and Mori, K. (1999). Mammalian transcription factor ATF6 is synthesized as a transmembrane protein and activated by proteolysis in response to endoplasmic reticulum stress. Mol. Biol. Cell 10, 3787-3799. doi: 10.1091/mbc.10.11.3787

Heman-Ackah, S. M., Manzano, R., Hoozemans, J. J. M., Scheper, W., Flynn, R., Haerty, W., et al. (2017). Alpha-synuclein induces the unfolded protein response in Parkinson's disease SNCA triplication iPSC-derived neurons. Hum. Mol. Genet. 26, 4441-4450. doi: 10.1093/hmg/ddx331

Hetz, C., and Papa, F. R. (2018). The unfolded protein response and cell fate control. Mol. Cell 69, 169-181. doi: 10.1016/j.molcel.2017.06.017

Hillary, R. F., and FitzGerald, U. (2018). A lifetime of stress: ATF6 in development and homeostasis. J. Biomed. Sci. 25:48. doi: 10.1186/s12929-0180453-1

Ho, D. H., Kim, H., Nam, D., Sim, H., Kim, J., Kim, H. G., et al. (2018). LRRK2 impairs autophagy by mediating phosphorylation of leucyl-tRNA synthetase. Cell Biochem. Funct. 36, 431-442. doi: 10.1002/cbf.3364

Ho, P. W., Leung, C. T., Liu, H., Pang, S. Y., Lam, C. S., Xian, J., et al. (2020). Agedependent accumulation of oligomeric SNCA/alpha-synuclein from impaired degradation in mutant LRRK2 knockin mouse model of Parkinson disease: role for therapeutic activation of chaperone-mediated autophagy (CMA). Autophagy 16, 347-370. doi: 10.1080/15548627.2019.1603545

Holtz, W. A., and O’Malley, K. L. (2003). Parkinsonian mimetics induce aspects of unfolded protein response in death of dopaminergic neurons. J. Biol. Chem. 278, 19367-19377. doi: 10.1074/jbc.M211821200

Hoozemans, J. J., van Haastert, E. S., Eikelenboom, P., de Vos, R. A., Rozemuller, J. M., and Scheper, W. (2007). Activation of the unfolded protein response in Parkinson's disease. Biochem. Biophys. Res. Commun. 354, 707-711. doi: 10.1016/j.bbrc.2007.01.043

Hoozemans, J. J., van Haastert, E. S., Nijholt, D. A., Rozemuller, A. J., and Scheper, W. (2012). Activation of the unfolded protein response is an early event in Alzheimer's and Parkinson's disease. Neurodegener. Dis. 10, 212-215. doi: 10. $1159 / 000334536$

Hou, X., Fiesel, F. C., Truban, D., Castanedes Casey, M., Lin, W. L., Soto, A. I., et al. (2018). Age- and disease-dependent increase of the mitophagy marker phospho-ubiquitin in normal aging and Lewy body disease. Autophagy 14, 1404-1418. doi: 10.1080/15548627.2018.1461294

Hou, X., Watzlawik, J. O., Fiesel, F. C., and Springer, W. (2020). Autophagy in Parkinson's disease. J. Mol. Biol. 432, 2651-2672. doi: 10.1016/j.jmb.2020. 01.037

Imai, Y., Soda, M., Inoue, H., Hattori, N., Mizuno, Y., and Takahashi, R. (2001). An unfolded putative transmembrane polypeptide, which can lead to endoplasmic reticulum stress, is a substrate of Parkin. Cell 105, 891-902. doi: 10.1016/s00928674(01)00407-x

Imai, Y., Soda, M., and Takahashi, R. (2000). Parkin suppresses unfolded protein stress-induced cell death through its E3 ubiquitin-protein ligase activity. J. Biol. Chem. 275, 35661-35664. doi: 10.1074/jbc.C000447200

Iurlaro, R., and Munoz-Pinedo, C. (2016). Cell death induced by endoplasmic reticulum stress. FEBS J. 283, 2640-2652. doi: 10.1111/febs.13598

Iwata, A., Maruyama, M., Kanazawa, I., and Nukina, N. (2001). alpha-Synuclein affects the MAPK pathway and accelerates cell death. J. Biol. Chem. 276, 45320-45329. doi: 10.1074/jbc.M103736200

Jiang, P., Gan, M., Ebrahim, A. S., Lin, W. L., Melrose, H. L., and Yen, S. H. (2010). ER stress response plays an important role in aggregation of alpha-synuclein. Mol. Neurodegener. 5:56. doi: 10.1186/1750-1326-5-56

Jiang, T. F., Zhang, Y. J., Zhou, H. Y., Wang, H. M., Tian, L. P., Liu, J., et al. (2013). Curcumin ameliorates the neurodegenerative pathology in A53T alphasynuclein cell model of Parkinson's disease through the downregulation of mTOR/p70S6K signaling and the recovery of macroautophagy. J. Neuroimmune Pharmacol. 8, 356-369. doi: 10.1007/s11481-012-9431-7

Jiao, F. J., Wang, Q. Z., Zhang, P., Yan, J. G., Zhang, Z., He, F., et al. (2017). CDK5mediated phosphorylation of XBP1s contributes to its nuclear translocation and activation in MPP(+)-induced Parkinson's disease model. Sci. Rep. 7:5622. doi: 10.1038/s41598-017-06012-6

Jovanovic-Tucovic, M., Harhaji-Trajkovic, L., Dulovic, M., Tovilovic-Kovacevic, G., Zogovic, N., Jeremic, M., et al. (2019). AMP-activated protein kinase inhibits MPP+-induced oxidative stress and apoptotic death of SH-SY5Y cells through sequential stimulation of Akt and autophagy. Eur. J. Pharmacol. 863:172677. doi: 10.1016/j.ejphar.2019.172677 
Kalia, L. V., and Lang, A. E. (2015). Parkinson's disease. Lancet 386, 896-912. doi: 10.1016/S0140-6736(14)61393-3

Kalogeropulou, A. F., Zhao, J., Bolliger, M. F., Memou, A., Narasimha, S., Molitor, T. P., et al. (2018). P62/SQSTM1 is a novel leucine-rich repeat kinase 2 (LRRK2) substrate that enhances neuronal toxicity. Biochem. J. 475, 1271-1293. doi: 10.1042/BCJ20170699

Kalvakolanu, D. V., and Gade, P. (2012). IFNG and autophagy: a critical role for the ER-stress mediator ATF6 in controlling bacterial infections. Autophagy 8, 1673-1674. doi: 10.4161/auto.21403

Karabiyik, C., Lee, M. J., and Rubinsztein, D. C. (2017). Autophagy impairment in Parkinson's disease. Essays Biochem. 61, 711-720. doi: 10.1042/EBC201 70023

Kitada, T., Asakawa, S., Hattori, N., Matsumine, H., Yamamura, Y., Minoshima, S., et al. (1998). Mutations in the parkin gene cause autosomal recessive juvenile parkinsonism. Nature 392, 605-608. doi: 10.1038/33416

Korennykh, A., and Walter, P. (2012). Structural basis of the unfolded protein response. Annu. Rev. Cell Dev. Biol. 28, 251-277. doi: 10.1146/annurev-cellbio101011-155826

Kouroku, Y., Fujita, E., Tanida, I., Ueno, T., Isoai, A., Kumagai, H., et al. (2007). ER stress (PERK/eIF2alpha phosphorylation) mediates the polyglutamine-induced LC3 conversion, an essential step for autophagy formation. Cell Death Differ. 14, 230-239. doi: 10.1038/sj.cdd.4401984

Lamark, T., Kirkin, V., Dikic, I., and Johansen, T. (2009). NBR1 and p62 as cargo receptors for selective autophagy of ubiquitinated targets. Cell Cycle 8, 1986-1990. doi: 10.4161/cc.8.13.8892

Ledesma, M. D., Galvan, C., Hellias, B., Dotti, C., and Jensen, P. H. (2002). Astrocytic but not neuronal increased expression and redistribution of parkin during unfolded protein stress. J. Neurochem. 83, 1431-1440. doi: 10.1046/j. 1471-4159.2002.01253.x

Lee, D. H., Kim, D., Kim, S. T., Jeong, S., Kim, J. L., Shim, S. M., et al. (2018). PARK7 modulates autophagic proteolysis through binding to the N-terminally arginylated form of the molecular chaperone HSPA5. Autophagy 14, 1870-1885. doi: 10.1080/15548627.2018.1491212

Lee, J. H., Han, J. H., Kim, H., Park, S. M., Joe, E. H., and Jou, I. (2019). Parkinson's disease-associated LRRK2-G2019S mutant acts through regulation of SERCA activity to control ER stress in astrocytes. Acta Neuropathol. Commun. 7:68. doi: 10.1186/s40478-019-0716-4

Lehtonen, S., Sonninen, T. M., Wojciechowski, S., Goldsteins, G., and Koistinaho, J. (2019). Dysfunction of cellular proteostasis in Parkinson's disease. Front. Neurosci. 13:457. doi: 10.3389/fnins.2019.00457

Levine, B., and Kroemer, G. (2019). Biological functions of autophagy genes: a disease perspective. Cell 176, 11-42. doi: 10.1016/j.cell.2018.09.048

Li, D. D., Wang, L. L., Deng, R., Tang, J., Shen, Y., Guo, J. F., et al. (2009). The pivotal role of c-Jun NH2-terminal kinase-mediated Beclin 1 expression during anticancer agents-induced autophagy in cancer cells. Oncogene 28, 886-898. doi: 10.1038/onc.2008.441

Li, L., and Hu, G. K. (2015). Pink1 protects cortical neurons from thapsigargininduced oxidative stress and neuronal apoptosis. Biosci. Rep. 35:e00174. doi: 10.1042/BSR20140104

Li, T., and Le, W. (2020). Biomarkers for Parkinson's disease: how good are they? Neurosci. Bull. 36, 183-194. doi: 10.1007/s12264-019-00433-1

Li, W., Zhu, J., Dou, J., She, H., Tao, K., Xu, H., et al. (2017). Phosphorylation of LAMP2A by p38 MAPK couples ER stress to chaperone-mediated autophagy. Nat. Commun. 8:1763. doi: 10.1038/s41467-017-01609-x

Lim, J., Kim, H. W., Youdim, M. B., Rhyu, I. J., Choe, K. M., and Oh, Y. J. (2011). Binding preference of p62 towards LC3-ll during dopaminergic neurotoxininduced impairment of autophagic flux. Autophagy 7, 51-60. doi: 10.4161/auto. 7.1.13909

Lim, J., Lee, Y., Jung, S., Youdim, M. B., and Oh, Y. J. (2014). Impaired autophagic flux is critically involved in drug-induced dopaminergic neuronal death. Parkinsonism Relat. Disord. 20(Suppl. 1), S162-S166. doi: 10.1016/ S1353-8020(13)70039-7

Liu, J., Liu, W., Li, R., and Yang, H. (2019). Mitophagy in Parkinson's disease: from pathogenesis to treatment. Cells 8:712. doi: $10.3390 /$ cells8070712

Liu, M., Qin, L., Wang, L., Tan, J., Zhang, H., Tang, J., et al. (2018). alphasynuclein induces apoptosis of astrocytes by causing dysfunction of the endoplasmic reticulumGolgi compartment. Mol. Med. Rep. 18, 322-332. doi: 10.3892/mmr. 2018.9002
Lucking, C. B., Durr, A., Bonifati, V., Vaughan, J., De Michele, G., Gasser, T., et al. (2000). Association between early-onset Parkinson's disease and mutations in the parkin gene. N. Engl. J. Med. 342, 1560-1567. doi: 10.1056/ NEJM200005253422103

Madureira, M., Connor-Robson, N., and Wade-Martins, R. (2020). LRRK2: Autophagy and Lysosomal activity. Front. Neurosci. 14:498. doi: 10.3389/fnins. 2020.00498

Malkus, K. A., and Ischiropoulos, H. (2012). Regional deficiencies in chaperone-mediated autophagy underlie alpha-synuclein aggregation and neurodegeneration. Neurobiol. Dis. 46, 732-744. doi: 10.1016/j.nbd.2012.03. 017

Manie, S. N., Lebeau, J., and Chevet, E. (2014). Cellular mechanisms of endoplasmic reticulum stress signaling in health and disease. 3. Orchestrating the unfolded protein response in oncogenesis: an update. Am. J. Physiol. Cell Physiol. 307, C901-C907. doi: 10.1152/ajpcell.00292.2014

Margariti, A., Li, H., Chen, T., Martin, D., Vizcay-Barrena, G., Alam, S., et al. (2013). XBP1 mRNA splicing triggers an autophagic response in endothelial cells through BECLIN-1 transcriptional activation. J. Biol. Chem. 288, 859-872. doi: $10.1074 /$ jbc.M112.412783

Martinez, A., Lopez, N., Gonzalez, C., and Hetz, C. (2019). Targeting of the unfolded protein response (UPR) as therapy for Parkinson's disease. Biol. Cell. 111, 161-168. doi: 10.1111/boc.201800068

Martinez-Vicente, M., Talloczy, Z., Kaushik, S., Massey, A. C., Mazzulli, J., Mosharov, E. V., et al. (2008). Dopamine-modified alpha-synuclein blocks chaperone-mediated autophagy. J. Clin. Invest. 118, 777-788. doi: 10.1172/ JCI32806

Martini-Stoica, H., Xu, Y., Ballabio, A., and Zheng, H. (2016). The AutophagyLysosomal pathway in neurodegeneration: a TFEB perspective. Trends Neurosci. 39, 221-234. doi: 10.1016/j.tins.2016.02.002

Meares, G. P., Hughes, K. J., Naatz, A., Papa, F. R., Urano, F., Hansen, P. A., et al. (2011). IRE1-dependent activation of AMPK in response to nitric oxide. Mol. Cell. Biol. 31, 4286-4297. doi: 10.1128/MCB.05668-11

Mencke, P., Boussaad, I., Romano, C. D., Kitami, T., Linster, C. L., and Kruger, R. (2021). The Role of DJ-1 in cellular metabolism and pathophysiological implications for Parkinson's disease. Cells 10:347. doi: 10.3390/cells10020347

Mercado, G., Castillo, V., Soto, P., Lopez, N., Axten, J. M., Sardi, S. P., et al. (2018). Targeting PERK signaling with the small molecule GSK2606414 prevents neurodegeneration in a model of Parkinson's disease. Neurobiol. Dis. 112, 136-148. doi: 10.1016/j.nbd.2018.01.004

Miyara, M., Kotake, Y., Tokunaga, W., Sanoh, S., and Ohta, S. (2016). Mild $\mathrm{MPP}(+)$ exposure impairs autophagic degradation through a novel lysosomal acidity-independent mechanism. J. Neurochem. 139, 294-308. doi: 10.1111/jnc. 13700

Moors, T. E., Hoozemans, J. J., Ingrassia, A., Beccari, T., Parnetti, L., ChartierHarlin, M. C., et al. (2017). Therapeutic potential of autophagy-enhancing agents in Parkinson's disease. Mol. Neurodegener. 12:11. doi: 10.1186/s13024017-0154-3

Moors, T. E., Paciotti, S., Ingrassia, A., Quadri, M., Breedveld, G., Tasegian, A., et al. (2019). Characterization of brain Lysosomal activities in GBA-related and sporadic Parkinson's disease and dementia with Lewy bodies. Mol. Neurobiol. 56, 1344-1355. doi: 10.1007/s12035-018-1090-0

Murphy, K. E., Gysbers, A. M., Abbott, S. K., Spiro, A. S., Furuta, A., Cooper, A., et al. (2015). Lysosomal-associated membrane protein 2 isoforms are differentially affected in early Parkinson's disease. Mov. Disord. 30, 1639-1647. doi: $10.1002 / \mathrm{mds} .26141$

Nakamura, S., and Yoshimori, T. (2017). New insights into autophagosomelysosome fusion. J. Cell Sci. 130, 1209-1216. doi: 10.1242/jcs.196352

Nascimbeni, A. C., Codogno, P., and Morel, E. (2017). Phosphatidylinositol-3phosphate in the regulation of autophagy membrane dynamics. FEBS J. 284, 1267-1278. doi: 10.1111/febs.13987

Nash, Y., Schmukler, E., Trudler, D., Pinkas-Kramarski, R., and Frenkel, D. (2017). DJ-1 deficiency impairs autophagy and reduces alpha-synuclein phagocytosis by microglia. J. Neurochem. 143, 584-594. doi: 10.1111/jnc.14222

Nguyen, T. N., Padman, B. S., and Lazarou, M. (2016). Deciphering the molecular signals of PINK1/Parkin mitophagy. Trends Cell Biol. 26, 733-744. doi: 10.1016/ j.tcb.2016.05.008

Ning, B., Zhang, Q., Deng, M., Wang, N., and Fang, Y. (2019a). Endoplasmic reticulum stress induced autophagy in 6-OHDA-induced Parkinsonian 
rats. Brain Res. Bull. 146, 224-227. doi: 10.1016/j.brainresbull.2019. 01.001

Ning, B., Zhang, Q., Wang, N., Deng, M., and Fang, Y. (2019b). beta-Asarone regulates er stress and autophagy via inhibition of the PERK/CHOP/Bcl2/Beclin-1 pathway in 6-OHDA-induced parkinsonian rats. Neurochem. Res. 44, 1159-1166. doi: 10.1007/s11064-019-02757-w

Noda, N. N., and Inagaki, F. (2015). Mechanisms of autophagy. Annu. Rev. Biophys. 44, 101-122. doi: 10.1146/annurev-biophys-060414-034248

Novoa, I., Zeng, H., Harding, H. P., and Ron, D. (2001). Feedback inhibition of the unfolded protein response by GADD34-mediated dephosphorylation of eIF2alpha. J. Cell Biol. 153, 1011-1022. doi: 10.1083/jcb.153.5.1011

Oakes, S. A., and Papa, F. R. (2015). The role of endoplasmic reticulum stress in human pathology. Annu. Rev. Pathol. 10, 173-194. doi: 10.1146/annurevpathol-012513-104649

Ogata, M., Hino, S., Saito, A., Morikawa, K., Kondo, S., Kanemoto, S., et al. (2006). Autophagy is activated for cell survival after endoplasmic reticulum stress. Mol. Cell. Biol. 26, 9220-9231. doi: 10.1128/MCB.01453-06

Orenstein, S. J., Kuo, S. H., Tasset, I., Arias, E., Koga, H., Fernandez-Carasa, I., et al. (2013). Interplay of LRRK2 with chaperone-mediated autophagy. Nat. Neurosci. 16, 394-406. doi: 10.1038/nn.3350

Paiva, I., Jain, G., Lazaro, D. F., Jercic, K. G., Hentrich, T., Kerimoglu, C., et al. (2018). Alpha-synuclein deregulates the expression of COL4A2 and impairs ER-Golgi function. Neurobiol. Dis. 119, 121-135. doi: 10.1016/j.nbd.2018.08. 001

Pandey, V. K., Mathur, A., and Kakkar, P. (2019). Emerging role of Unfolded Protein Response (UPR) mediated proteotoxic apoptosis in diabetes. Life Sci. 216, 246-258. doi: 10.1016/j.lfs.2018.11.041

Park, S., Han, S., Choi, I., Kim, B., Park, S. P., Joe, E. H., et al. (2016). Interplay between Leucine-rich repeat Kinase 2 (LRRK2) and p62/SQSTM-1 in selective Autophagy. PLoS One 11:e0163029. doi: 10.1371/journal.pone.0163029

Pattingre, S., Tassa, A., Qu, X., Garuti, R., Liang, X. H., Mizushima, N., et al. (2005). Bcl-2 antiapoptotic proteins inhibit Beclin 1-dependent autophagy. Cell 122, 927-939. doi: 10.1016/j.cell.2005.07.002

Poewe, W., Seppi, K., Tanner, C. M., Halliday, G. M., Brundin, P., Volkmann, J., et al. (2017). Parkinson disease. Nat. Rev. Dis. Primers 3:17013. doi: 10.1038/ nrdp.2017.13

Puschmann, A. (2013). Monogenic Parkinson's disease and parkinsonism: clinical phenotypes and frequencies of known mutations. Parkinsonism Relat. Disord. 19, 407-415. doi: 10.1016/j.parkreldis.2013.01.020

Ramalingam, M., Huh, Y. J., and Lee, Y. I. (2019). The impairments of alphaSynuclein and mechanistic target of rapamycin in rotenone-induced SH-SY5Y cells and mice model of Parkinson's disease. Front. Neurosci. 13:1028. doi: $10.3389 /$ fnins. 2019.01028

Rashid, H. O., Yadav, R. K., Kim, H. R., and Chae, H. J. (2015). ER stress: autophagy induction, inhibition and selection. Autophagy 11, 1956-1977. doi: 10.1080/ 15548627.2015.1091141

Ren, H., Fu, K., Mu, C., Li, B., Wang, D., and Wang, G. (2010). DJ-1, a cancer and Parkinson's disease associated protein, regulates autophagy through JNK pathway in cancer cells. Cancer Lett. 297, 101-108. doi: 10.1016/j.canlet.2010. 05.001

Rouschop, K. M., van den Beucken, T., Dubois, L., Niessen, H., Bussink, J., Savelkouls, K., et al. (2010). The unfolded protein response protects human tumor cells during hypoxia through regulation of the autophagy genes MAP1LC3B and ATG5. J. Clin. Invest. 120, 127-141. doi: 10.1172/JCI40027

Rubinsztein, D. C., and Nixon, R. A. (2010). Rapamycin induces autophagic flux in neurons. Proc. Natl. Acad. Sci. U.S.A. 107:E181. doi: 10.1073/pnas.1014633107

Russell, R. C., Tian, Y., Yuan, H., Park, H. W., Chang, Y. Y., Kim, J., et al. (2013). ULK1 induces autophagy by phosphorylating Beclin-1 and activating VPS34 lipid kinase. Nat. Cell Biol. 15, 741-750. doi: 10.1038/ncb2757

Ryu, E. J., Harding, H. P., Angelastro, J. M., Vitolo, O. V., Ron, D., and Greene, L. A. (2002). Endoplasmic reticulum stress and the unfolded protein response in cellular models of Parkinson's disease. J. Neurosci. 22, 10690-10698.

Rzymski, T., Milani, M., Pike, L., Buffa, F., Mellor, H. R., Winchester, L., et al. (2010). Regulation of autophagy by ATF4 in response to severe hypoxia. Oncogene 29, 4424-4435. doi: 10.1038/onc.2010.191

Sado, M., Yamasaki, Y., Iwanaga, T., Onaka, Y., Ibuki, T., Nishihara, S., et al. (2009). Protective effect against Parkinson's disease-related insults through the activation of XBP1. Brain Res. 1257, 16-24. doi: 10.1016/j.brainres.2008.11.104
Salazar, M., Carracedo, A., Salanueva, I. J., Hernandez-Tiedra, S., Lorente, M., Egia, A., et al. (2009). Cannabinoid action induces autophagy-mediated cell death through stimulation of ER stress in human glioma cells. J. Clin. Invest. 119, 1359-1372. doi: $10.1172 /$ jci37948

Selvaraj, S., Sun, Y., Watt, J. A., Wang, S., Lei, S., Birnbaumer, L., et al. (2012). Neurotoxin-induced ER stress in mouse dopaminergic neurons involves downregulation of TRPC1 and inhibition of AKT/mTOR signaling. J. Clin. Invest. 122, 1354-1367. doi: 10.1172/JCI61332

Senft, D., and Ronai, Z. A. (2015). UPR, autophagy, and mitochondria crosstalk underlies the ER stress response. Trends Biochem. Sci. 40, 141-148. doi: 10.1016/ j.tibs.2015.01.002

Shahmoradian, S. H., Lewis, A. J., Genoud, C., Hench, J., Moors, T. E., Navarro, P. P., et al. (2019). Lewy pathology in Parkinson's disease consists of crowded organelles and lipid membranes. Nat. Neurosci. 22, 1099-1109. doi: 10.1038/ s41593-019-0423-2

Shen, J., Chen, X., Hendershot, L., and Prywes, R. (2002). ER stress regulation of ATF6 localization by dissociation of BiP/GRP78 binding and unmasking of Golgi localization signals. Dev. Cell 3, 99-111. doi: 10.1016/s1534-5807(02) 00203-4

Shimizu, S. (2018). Biological roles of alternative Autophagy. Mol. Cells 41, 50-54. doi: 10.14348/molcells.2018.2215

Shimura, H., Hattori, N., Kubo, S., Mizuno, Y., Asakawa, S., Minoshima, S., et al. (2000). Familial Parkinson disease gene product, parkin, is a ubiquitin-protein ligase. Nat. Genet. 25, 302-305. doi: 10.1038/77060

Shulman, J. M., De Jager, P. L., and Feany, M. B. (2011). Parkinson's disease: genetics and pathogenesis. Annu. Rev. Pathol. 6, 193-222. doi: 10.1146/ annurev-pathol-011110-130242

Simola, N., Morelli, M., and Carta, A. R. (2007). The 6-hydroxydopamine model of Parkinson's disease. Neurotox. Res. 11, 151-167. doi: 10.1007/BF03033565

Singh, K., Han, K., Tilve, S., Wu, K., Geller, H. M., and Sack, M. N. (2018). Parkin targets NOD2 to regulate astrocyte endoplasmic reticulum stress and inflammation. Glia 66, 2427-2437. doi: 10.1002/glia.23482

Slodzinski, H., Moran, L. B., Michael, G. J., Wang, B., Novoselov, S., Cheetham, M. E., et al. (2009). Homocysteine-induced endoplasmic reticulum protein (herp) is up-regulated in parkinsonian substantia nigra and present in the core of Lewy bodies. Clin. Neuropathol. 28, 333-343.

Smith, W. W., Jiang, H., Pei, Z., Tanaka, Y., Morita, H., Sawa, A., et al. (2005). Endoplasmic reticulum stress and mitochondrial cell death pathways mediate A53T mutant alpha-synuclein-induced toxicity. Hum. Mol. Genet. 14, 38013811. doi: $10.1093 / \mathrm{hmg} / \mathrm{ddi} 396$

Song, J. X., Lu, J. H., Liu, L. F., Chen, L. L., Durairajan, S. S., Yue, Z., et al. (2014). HMGB1 is involved in autophagy inhibition caused by SNCA/alphasynuclein overexpression: a process modulated by the natural autophagy inducer corynoxine B. Autophagy 10, 144-154. doi: 10.4161/auto.26751

Stefanis, L., Larsen, K. E., Rideout, H. J., Sulzer, D., and Greene, L. A. (2001). Expression of A53T mutant but not wild-type alpha-synuclein in PC12 cells induces alterations of the ubiquitin-dependent degradation system, loss of dopamine release, and autophagic cell death. J. Neurosci. 21, 9549-9560.

Stolz, A., Ernst, A., and Dikic, I. (2014). Cargo recognition and trafficking in selective autophagy. Nat. Cell Biol. 16, 495-501. doi: 10.1038/ncb2979

Sugeno, N., Takeda, A., Hasegawa, T., Kobayashi, M., Kikuchi, A., Mori, F., et al. (2008). Serine 129 phosphorylation of alpha-synuclein induces unfolded protein response-mediated cell death. J. Biol. Chem. 283, 23179-23188. doi: 10.1074/jbc.M802223200

Swerdlow, N. S., and Wilkins, H. M. (2020). Mitophagy and the brain. Int. J. Mol. Sci. 21:9661. doi: 10.3390/ijms21249661

Takahashi, Y., He, H., Tang, Z., Hattori, T., Liu, Y., Young, M. M., et al. (2018). An autophagy assay reveals the ESCRT-III component CHMP2A as a regulator of phagophore closure. Nat. Commun. 9:2855. doi: 10.1038/s41467-018-05254-w

Takahashi, Y., Liang, X., Hattori, T., Tang, Z., He, H., Chen, H., et al. (2019). VPS37A directs ESCRT recruitment for phagophore closure. J. Cell Biol. 218, 3336-3354. doi: 10.1083/jcb.201902170

Tanji, K., Mori, F., Kakita, A., Takahashi, H., and Wakabayashi, K. (2011). Alteration of autophagosomal proteins (LC3, GABARAP and GATE-16) in Lewy body disease. Neurobiol. Dis. 43, 690-697. doi: 10.1016/j.nbd.2011.05.022

Tekirdag, K., and Cuervo, A. M. (2018). Chaperone-mediated autophagy and endosomal microautophagy: joint by a chaperone. J. Biol. Chem. 293, 54145424. doi: $10.1074 /$ jbc.R117.818237 
Tolosa, E., Vila, M., Klein, C., and Rascol, O. (2020). LRRK2 in Parkinson disease: challenges of clinical trials. Nat. Rev. Neurol. 16, 97-107. doi: 10.1038/s41582019-0301-2

Tong, Q., Wu, L., Gao, Q., Ou, Z., Zhu, D., and Zhang, Y. (2016a). PPARbeta/delta agonist provides neuroprotection by suppression of IRE1alpha-caspase-12mediated endoplasmic reticulum stress pathway in the rotenone rat model of Parkinson's disease. Mol. Neurobiol. 53, 3822-3831. doi: 10.1007/s12035-0159309-9

Tong, Q., Wu, L., Jiang, T., Ou, Z., Zhang, Y., and Zhu, D. (2016b). Inhibition of endoplasmic reticulum stress-activated IRE1alpha-TRAF2 caspase-12 apoptotic pathway is involved in the neuroprotective effects of telmisartan in the rotenone rat model of Parkinson's disease. Eur. J. Pharmacol. 776, 106-115. doi: 10.1016/j.ejphar.2016.02.042

Torres-Odio, S., Key, J., Hoepken, H. H., Canet-Pons, J., Valek, L., Roller, B., et al. (2017). Progression of pathology in PINK1-deficient mouse brain from splicing via ubiquitination, ER stress, and mitophagy changes to neuroinflammation. J. Neuroinflamm. 14:154. doi: 10.1186/s12974-017-0928-0

Toyofuku, T., Okamoto, Y., Ishikawa, T., Sasawatari, S., and Kumanogoh, A. (2020). LRRK2 regulates endoplasmic reticulum-mitochondrial tethering through the PERK-mediated ubiquitination pathway. EMBO J. 39:e100875. doi: $10.15252 / \mathrm{embj} .2018100875$

Tsuboyama, K., Koyama-Honda, I., Sakamaki, Y., Koike, M., Morishita, H., and Mizushima, N. (2016). The ATG conjugation systems are important for degradation of the inner autophagosomal membrane. Science 354, 1036-1041. doi: 10.1126/science.aaf6136

Turano, C., Coppari, S., Altieri, F., and Ferraro, A. (2002). Proteins of the PDI family: unpredicted non-ER locations and functions. J. Cell. Physiol. 193, 154-163. doi: 10.1002/jcp.10172

Urano, F., Wang, X., Bertolotti, A., Zhang, Y., Chung, P., Harding, H. P., et al. (2000). Coupling of stress in the ER to activation of JNK protein kinases by transmembrane protein kinase IRE1. Science 287, 664-666. doi: 10.1126/ science.287.5453.664

Valdes, P., Mercado, G., Vidal, R. L., Molina, C., Parsons, G., Court, F. A., et al. (2014). Control of dopaminergic neuron survival by the unfolded protein response transcription factor XBP1. Proc. Natl. Acad. Sci. U.S.A. 111, 68046809. doi: 10.1073/pnas.1321845111

Valente, E. M., Abou-Sleiman, P. M., Caputo, V., Muqit, M. M., Harvey, K., Gispert, S., et al. (2004a). Hereditary early-onset Parkinson's disease caused by mutations in PINK1. Science 304, 1158-1160. doi: 10.1126/science.1096284

Valente, E. M., Salvi, S., Ialongo, T., Marongiu, R., Elia, A. E., Caputo, V., et al. (2004b). PINK1 mutations are associated with sporadic early-onset parkinsonism. Ann. Neurol. 56, 336-341. doi: 10.1002/ana.20256

Verfaillie, T., Salazar, M., Velasco, G., and Agostinis, P. (2010). Linking ER stress to autophagy: potential implications for cancer therapy. Int. J. Cell Biol. 2010:930509. doi: 10.1155/2010/930509

Vidal, R. L., Figueroa, A., Court, F. A., Thielen, P., Molina, C., Wirth, C., et al. (2012). Targeting the UPR transcription factor XBP1 protects against Huntington's disease through the regulation of FoxO1 and autophagy. Hum. Mol. Genet. 21, 2245-2262. doi: 10.1093/hmg/dds040

Vitte, J., Traver, S., Maues De Paula, A., Lesage, S., Rovelli, G., Corti, O., et al. (2010). Leucine-rich repeat kinase 2 is associated with the endoplasmic reticulum in dopaminergic neurons and accumulates in the core of Lewy bodies in Parkinson disease. J. Neuropathol. Exp. Neurol. 69, 959-972. doi: 10.1097/NEN. 0b013e3181efc01c

Walter, P., and Ron, D. (2011). The unfolded protein response: from stress pathway to homeostatic regulation. Science 334, 1081-1086. doi: 10.1126/ science. 1209038

Wang, M., and Kaufman, R. J. (2016). Protein misfolding in the endoplasmic reticulum as a conduit to human disease. Nature 529, 326-335. doi: 10.1038/ nature 17041

Wang, R., Sun, H., Ren, H., and Wang, G. (2020a). alpha-Synuclein aggregation and transmission in Parkinson's disease: a link to mitochondria and lysosome. Sci. China Life Sci. 63, 1850-1859. doi: 10.1007/s11427-020-1756-9

Wang, R., Sun, H., Wang, G., and Ren, H. (2020b). Imbalance of Lysine Acetylation contributes to the pathogenesis of Parkinson's disease. Int. J. Mol. Sci. 21:7182. doi: 10.3390/ijms 21197182

Wang, X., Zhai, H., and Wang, F. (2018). 6-OHDA induces oxidation of F-box protein Fbw7beta by chaperone-mediated Autophagy in
Parkinson's model. Mol. Neurobiol. 55, 4825-4833. doi: 10.1007/s12035-0170686-0

Wei, Y., Pattingre, S., Sinha, S., Bassik, M., and Levine, B. (2008). JNK1-mediated phosphorylation of Bcl-2 regulates starvation-induced autophagy. Mol. Cell 30, 678-688. doi: 10.1016/j.molcel.2008.06.001

Weidberg, H., Shvets, E., Shpilka, T., Shimron, F., Shinder, V., and Elazar, Z. (2010). LC3 and GATE-16/GABARAP subfamilies are both essential yet act differently in autophagosome biogenesis. EMBO J. 29, 1792-1802. doi: 10.1038/emboj. 2010.74

Winslow, A. R., Chen, C. W., Corrochano, S., Acevedo-Arozena, A., Gordon, D. E., Peden, A. A., et al. (2010). alpha-Synuclein impairs macroautophagy: implications for Parkinson's disease. J. Cell Biol. 190, 1023-1037. doi: 10.1083/ jcb.201003122

Wu, F., Xu, H. D., Guan, J. J., Hou, Y. S., Gu, J. H., Zhen, X. C., et al. (2015). Rotenone impairs autophagic flux and lysosomal functions in Parkinson's disease. Neuroscience 284, 900-911. doi: 10.1016/j.neuroscience.2014. 11.004

Wu, L., Luo, N., Zhao, H. R., Gao, Q., Lu, J., Pan, Y., et al. (2014). Salubrinal protects against rotenone-induced SH-SY5Y cell death via ATF4-parkin pathway. Brain Res. 1549, 52-62. doi: 10.1016/j.brainres.2014.01.003

Xie, L., Tiong, C. X., and Bian, J. S. (2012). Hydrogen sulfide protects SH-SY5Y cells against 6-hydroxydopamine-induced endoplasmic reticulum stress. Am. J. Physiol. Cell Physiol. 303, C81-C91. doi: 10.1152/ajpcell.00281.2011

Xilouri, M., Vogiatzi, T., Vekrellis, K., Park, D., and Stefanis, L. (2009). Abberant alpha-synuclein confers toxicity to neurons in part through inhibition of chaperone-mediated autophagy. PLoS One 4:e5515. doi: 10.1371/journal.pone. 0005515

Xiong, N., Xiong, J., Jia, M., Liu, L., Zhang, X., Chen, Z., et al. (2013). The role of autophagy in Parkinson's disease: rotenone-based modeling. Behav. Brain Funct. 9:13. doi: 10.1186/1744-9081-9-13

Xu, C. Y., Kang, W. Y., Chen, Y. M., Jiang, T. F., Zhang, J., Zhang, L. N., et al. (2017). DJ-1 inhibits alpha-synuclein aggregation by regulating chaperone-mediated Autophagy. Front. Aging Neurosci. 9:308. doi: 10.3389/fnagi.2017.00308

Xu, J., Zhou, Q., Xu, W., and Cai, L. (2012). Endoplasmic reticulum stress and diabetic cardiomyopathy. Exp. Diabetes Res. 2012:827971. doi: 10.1155/2012/ 827971

Yamamoto, K., Sato, T., Matsui, T., Sato, M., Okada, T., Yoshida, H., et al. (2007). Transcriptional induction of mammalian ER quality control proteins is mediated by single or combined action of ATF6alpha and XBP1. Dev. Cell 13, 365-376. doi: 10.1016/j.devcel.2007.07.018

Yamamuro, A., Yoshioka, Y., Ogita, K., and Maeda, S. (2006). Involvement of endoplasmic reticulum stress on the cell death induced by 6-hydroxydopamine in human neuroblastoma SH-SY5Y cells. Neurochem. Res. 31, 657-664. doi: 10.1007/s11064-006-9062-6

Yang, J., Kim, K. S., Iyirhiaro, G. O., Marcogliese, P. C., Callaghan, S. M., Qu, D., et al. (2019). DJ-1 modulates the unfolded protein response and cell death via upregulation of ATF4 following ER stress. Cell Death Dis. 10:135. doi: 10.1038/ s41419-019-1354-2

Yokota, T., Sugawara, K., Ito, K., Takahashi, R., Ariga, H., and Mizusawa, H. (2003). Down regulation of DJ-1 enhances cell death by oxidative stress, ER stress, and proteasome inhibition. Biochem. Biophys. Res. Commun. 312, 1342-1348. doi: 10.1016/j.bbrc.2003.11.056

Yorimitsu, T., Nair, U., Yang, Z., and Klionsky, D. J. (2006). Endoplasmic reticulum stress triggers autophagy. J. Biol. Chem. 281, 30299-30304. doi: 10.1074/jbc. M607007200

Young, M. M., and Wang, H. G. (2018). Sphingolipids as regulators of Autophagy and Endocytic trafficking. Adv. Cancer Res. 140, 27-60. doi: 10.1016/bs.acr. 2018.04.008

Yu, W. H., Dorado, B., Figueroa, H. Y., Wang, L., Planel, E., Cookson, M. R., et al. (2009). Metabolic activity determines efficacy of macroautophagic clearance of pathological oligomeric alpha-synuclein. Am. J. Pathol. 175, 736-747. doi: 10.2353/ajpath.2009.080928

Yuan, Y., Cao, P., Smith, M. A., Kramp, K., Huang, Y., Hisamoto, N., et al. (2011). Dysregulated LRRK2 signaling in response to endoplasmic reticulum stress leads to dopaminergic neuron degeneration in C. elegans. PLoS One 6:e22354. doi: 10.1371/journal.pone.0022354

Yung, H. W., Charnock-Jones, D. S., and Burton, G. J. (2011). Regulation of AKT phosphorylation at Ser473 and Thr308 by endoplasmic reticulum stress 
modulates substrate specificity in a severity dependent manner. PLoS One 6:e17894. doi: 10.1371/journal.pone.0017894

Zalckvar, E., Berissi, H., Mizrachy, L., Idelchuk, Y., Koren, I., Eisenstein, M., et al. (2009). DAP-kinase-mediated phosphorylation on the BH3 domain of beclin 1 promotes dissociation of beclin 1 from Bcl-XL and induction of autophagy. EMBO Rep. 10, 285-292. doi: 10.1038/embor.2008.246

Zhang, X., Yuan, Y., Jiang, L., Zhang, J., Gao, J., Shen, Z., et al. (2014). Endoplasmic reticulum stress induced by tunicamycin and thapsigargin protects against transient ischemic brain injury: involvement of PARK2-dependent mitophagy. Autophagy 10, 1801-1813. doi: 10.4161/auto.32136

Zhao, Y., Li, X., Cai, M. Y., Ma, K., Yang, J., Zhou, J., et al. (2013). XBP-1u suppresses autophagy by promoting the degradation of FoxO1 in cancer cells. Cell Res. 23, 491-507. doi: 10.1038/cr.2013.2

Zhao, Y. G., Chen, Y., Miao, G., Zhao, H., Qu, W., Li, D., et al. (2017). The ERLocalized transmembrane protein EPG-3/VMP1 regulates SERCA activity to control ER-isolation membrane contacts for Autophagosome formation. Mol. Cell 67, 974-989.e976. doi: 10.1016/j.molcel.2017.08.005

Zhao, Y. G., and Zhang, H. (2019). Autophagosome maturation: an epic journey from the ER to lysosomes. J. Cell Biol. 218, 757-770. doi: 10.1083/jcb.201810099

Zhou, Y., Lee, J., Reno, C. M., Sun, C., Park, S. W., Chung, J., et al. (2011). Regulation of glucose homeostasis through a XBP-1-FoxO1 interaction. Nat. Med. 17, 356-365. doi: 10.1038/nm.2293

Zhu, J. H., Guo, F., Shelburne, J., Watkins, S., and Chu, C. T. (2003). Localization of phosphorylated ERK/MAP kinases to mitochondria and autophagosomes in Lewy body diseases. Brain Pathol. 13, 473-481. doi: 10.1111/j.1750-3639.2003. tb00478.x
Zhu, J. H., Gusdon, A. M., Cimen, H., Van Houten, B., Koc, E., and Chu, C. T. (2012). Impaired mitochondrial biogenesis contributes to depletion of functional mitochondria in chronic MPP+ toxicity: dual roles for ERK1/2. Cell Death Dis. 3:e312. doi: 10.1038/cddis.2012.46

Zhu, J. H., Horbinski, C., Guo, F., Watkins, S., Uchiyama, Y., and Chu, C. T. (2007). Regulation of autophagy by extracellular signal-regulated protein kinases during 1-methyl-4-phenylpyridinium-induced cell death. Am. J. Pathol. 170, 75-86. doi: 10.2353/ajpath.2007.060524

Zhu, X., Huang, L., Gong, J., Shi, C., Wang, Z., Ye, B., et al. (2017). NFkappaB pathway link with ER stress-induced autophagy and apoptosis in cervical tumor cells. Cell Death Discov. 3:17059. doi: 10.1038/cddiscovery. 2017.59

Zhu, Y., Wang, C., Yu, M., Cui, J., Liu, L., and Xu, Z. (2013). ULK1 and JNK are involved in mitophagy incurred by LRRK2 G2019S expression. Protein Cell 4, 711-721. doi: 10.1007/s13238-013-3910-3

Conflict of Interest: The authors declare that the research was conducted in the absence of any commercial or financial relationships that could be construed as a potential conflict of interest.

Copyright (c) 2021 Ren, Zhai, Lu and Wang. This is an open-access article distributed under the terms of the Creative Commons Attribution License (CC BY). The use, distribution or reproduction in other forums is permitted, provided the original author(s) and the copyright owner(s) are credited and that the original publication in this journal is cited, in accordance with accepted academic practice. No use, distribution or reproduction is permitted which does not comply with these terms. 